Pacific Journal of Mathematics

A MATRIX VOLTERRA INTEGRODIFFERENTIAL EQUATION
OCCURRING IN POLYMER RHEOLOGY 


\title{
A MATRIX VOLTERRA INTEGRODIFFERENTIAL EQUATION OCCURRING IN POLYMER RHEOLOGY
}

\author{
HANS ENGLER
}

\begin{abstract}
In this paper we study a class of Volterra integrodifferential equations that arise in the description of elastic liquids, such as polymer melts and dilute or concentrated polymer solutions. The deformation of a small cube-shaped sample of such a liquid can be approximately described by a symmetric $3 \times 3$-matrix: If the material undergoes some prescribed deformation for times $t \leq 0$ and then is allowed to recover without constraints for $t>0$ (stress-free recoil), and if inertial effects are ignored, these matrices obey an ordinary first order Volterra integrodifferential equation. Incompressibility of the material imposes the nonlinear constraint that the determinant of the matrices remain constant. In addition, there is a natural small parameter $\eta>0$, proportional to Newtonian viscosity, which multiplies the derivative. In the case $\eta=0$, which is also of physical interest, the problem reduces to an implicit Volterra integral equation.
\end{abstract}

1. Introduction. The problem under study thus can be classified as a singularly perturbed Volterra integrodifferential equation on a manifold. In this paper we present an existence and uniqueness theory, asymptotic results (as $t \rightarrow \infty$ ) for the cases $\eta>0$ and $\eta=0$, and a study of the behavior of the solutions as $\eta \downarrow 0$. Thus, we follow the program of the influential paper [11], in which these questions were studied for the case of elongational flows.

In the remainder of this section, the physical background of the problem is described, the equations to be studied are derived, and some special classes of deformations are listed for later reference. Section 2 reviews known results for the important class of elongational deformations. In $\S 3$, we develop some useful facts concerning the differential geometry of the manifold on which the equation holds. In $\S 4$, we show that the problem under study has a natural gradient structure and give a basic local existence and uniqueness theorem for the case $\eta>0$. Section 5 deals with global existence and with the existence of asymptotic limits of solutions as $t \rightarrow \infty$, still for $\eta>0$. In $\S 6$, we develop a variational framework in which local and global existence and uniqueness and the existence of asymptotic limits can also be proved for the reduced implicit equation that results from setting 
$\eta=0$. In $\S 7$, we study the behavior of solutions as $\eta \downarrow 0$, in an initial layer near $t=0$. Section 8 is concerned with this question for large $t$. The results of these two sections give a description up to terms of order $\eta^{\alpha}$ for some $\alpha>0$, uniformly in $0 \leq t<\infty$. In $\S 9$, some general qualitative properties of asymptotic limits are presented, and these results are used in $\S 10$ to give a unified explanation for various free recoil phenomena such as non-recovery of the initial shape after a stretching history and lateral expansion after a previous simple shear.

If the material under consideration occupies a reference region $\Omega \subset$ $\mathbf{R}^{3}$ at time $t=-\infty$, its deformation can be described by a family of diffeomorphisms

$$
\mathbf{y}(\cdot, t): \Omega \rightarrow \mathbf{R}^{3} \quad(t \in \mathbf{R}) .
$$

Let

$$
\mathbf{F}(\xi, t)=\left(\frac{\partial \mathbf{y}_{i}(\xi, t)}{\partial \xi_{j}}\right)_{i, j}
$$

denote the deformation gradient, and let

$$
\Pi(\cdot, t): \Omega \rightarrow \mathbf{R}^{3,3} \quad(t \in \mathbf{R})
$$

denote the body stress tensor (see [10]). Then isochoric motions of the material are governed by the partial differential equations

$$
\rho\left(\frac{\partial^{2} \mathbf{y}(\xi, t)}{\partial^{2} t}-\mathbf{f}(\xi, t)\right)=\nabla_{\xi} \cdot(\mathbf{F}(\xi, t) \Pi(\xi, t)),
$$

where $\rho$ is the mass density, assumed to be constant, $f$ denotes the body force per unit mass, and the divergence is formed with respect to the second component (cf. [5], [10], [17]). Here $\Pi(\xi, t)$ depends on the deformation gradient (and its history up to time $t$ ) at the particle $\xi$ through a suitable constitutive relation. For isochoric motions, we have to add the incompressibility condition

$$
\operatorname{det} \mathbf{F}(\xi, t)=1 \text { for all }(\xi, t) \text {. }
$$

This paper is concerned with homogeneous flows, i.e. flows for which $\mathbf{F}$ does not depend on $\xi$. Thus $\mathbf{y}(\cdot, t)$ should be an affine mapping for all $t \in \mathbf{R}$, and the equations of motion (2) will only hold for very special body forces. Heuristically, the "interior" behavior of the material (away from any boundary effects) might be described in this way. The class of experiments to be described here consists in subjecting the material to a homogeneous deformation up to time $t=0$ and then allowing its stress-free recovery for $t>0$ with vanishing 
body forces. The analysis below is based on the additional simplifying assumption that the inertial forces can be neglected for $t>0$; thus the equations of motion will no longer be satisfied. Nevertheless, such simplifications can be used to test the applicability of constitutive relations by comparing mathematical predictions to experimental observations; see [9].

The mathematical problem thus consists in solving the implicit equation for $\mathbf{F}(\cdot)$ (the dependence on $\xi$ can now be omitted)

$$
\Pi(t)=0 \quad(t>0)
$$

for a given history $(\mathbf{F}(t))_{t \leq 0}$. A relation between $\Pi(t)$ and the values of $(\mathbf{F}(s))_{s \leq t}$ then is needed to close the system of equations; a general class of such relations will now be introduced.

Let $\gamma(t)=\mathbf{F}^{T}(t) \mathbf{F}(t)$ denote Green's deformation tensor. The KBKZ-model for incompressible elastic liquids (proposed in [2] and [8]) postulates a relation of the form

$$
\text { (5) } \begin{aligned}
\Pi(t)= & -p(t) \gamma^{-1}(t)-\eta \frac{d}{d t} \gamma^{-1}(t) \\
& +\int_{-\infty}^{t}\left(W_{1}\left(t-s, \lambda_{1}(t, s), \lambda_{2}(t, s)\right) \gamma^{-1}(s)\right. \\
& \left.\quad-W_{2}\left(t-s, \lambda_{1}(t, s), \lambda_{2}(t, s)\right) \gamma^{-1}(t) \gamma(s) \gamma^{-1}(t)\right) d t,
\end{aligned}
$$

where $p(\cdot)$ is a reactive scalar pressure, $\eta \geq 0$ is Newtonian viscosity, $\lambda_{1}(t, s)=\operatorname{tr}\left(\gamma(t) \gamma^{-1}(s)\right)$ and $\gamma_{2}(t, s)=\operatorname{tr}\left(\gamma^{-1}(t) \gamma(s)\right)$ are relative strain invariants, and

$$
W_{i}\left(\tau, \lambda_{1}, \lambda_{2}\right)=\frac{\partial W}{\partial \lambda_{i}}\left(\tau, \lambda_{1}, \lambda_{2}\right)
$$

for a suitable scalar function $W:(0, \infty) \times[3, \infty) \times[3, \infty) \rightarrow \mathbf{R}$. The constitutive relation (5) contains the case of a Newtonian liquid (for $W=0$ ) and is analogous to the well-known formula for incompressible isotropic homogeneous hyperelastic materials ([5], [19]). It can be specialized to many other constitutive laws of integral type (see $\S 10$ below).

The problem of free elastic recoil for homogeneous flows therefore consists in finding a solution $(\gamma(\cdot), p(\cdot))$ of $(4),(5)$, together with the incompressibility condition

$$
\operatorname{det} \gamma(\cdot)=1,
$$

for prescribed $\gamma(\cdot)$ on $(-\infty, 0]$ that satisfies also $(6)$. 
The components of $\gamma$ and of its inverse $\gamma^{-1}$ describe the deformation of an embedded material sample that is a parallelopiped of unit volume, in the following way. Let $e_{1}, e_{2}, e_{3}$ denote the vectors describing the edges of the parallelopiped. The lengths of these edges at time $t$ are obviously given by $\sqrt{\gamma_{i i}}$, and cosines of angles between edges are given by $\gamma_{i j} / \sqrt{\gamma_{i i} \gamma_{j j}}$, if $i \neq j$. Let $E_{i j}, E_{i j}^{\prime}$ denote the two faces of the parallelopiped that are parallel to $e_{i}$ and $e_{j} \quad(i \neq j)$, let $\gamma^{-1}=\left(\gamma^{i j}\right)_{i, j}$, and set $k=6-i-j$. Then the separation between $E_{i j}$ and $E_{i j}^{\prime}$ at time $t$ is equal to $\left(\gamma^{k k}(t)\right)^{-1 / 2}$, and the cosine of the angle between $E_{i j}$ and $E_{k j}$ is given by $\gamma^{i k}(t) / \sqrt{\gamma^{i i}(t) \gamma^{k k}(t)}$.

Certain deformations are of special interest, such as shear-free deformations, where

$$
\gamma=\left(\begin{array}{lll}
a & 0 & 0 \\
0 & b & 0 \\
0 & 0 & c
\end{array}\right) .
$$

Here and below all quantities depend on $t$. Examples of such deformations are filament stretching, where $a=y \geq 1, b=c=y^{-1 / 2}$, sheet stretching, which is of the same form with $y \leq 1$, and a deformation called pure shear (rather inappropriately), where $a=x, b=$ $x^{-1}, c=1$ and which describes e.g. a deformation between two rolls. In the case of filament stretching, the quantity $y$ is called the elongation ratio. Another important class consists in shear flows in which

$$
\gamma=\left(\begin{array}{ccc}
1 & a & b \\
a & 1+a^{2} & a b+c \\
b & a b+c & 1+b^{2}+c^{2}
\end{array}\right),
$$

with $a, b, c \in \mathbf{R}$. In particular, flows in which $a(\cdot)=s(\cdot)$ and $b(\cdot)=c(\cdot)=0$ are called simple shear flows, and in this case $\theta(t)=$ $\arctan s(t)$ is known as the angle of shear. It will be shown in $\S 10$ that flow histories of the form (7) will give deformations of the same form under free recovery and that in general flow histories of the form (8) will not result in deformations of the same form under free recovery.

For later reference we define the set

$$
\mathbf{M}=\left\{A \in \mathbf{R}^{3,3} \mid A=A^{T}, \operatorname{det} A=1, A>0\right\} .
$$

The unit matrix will be denoted by $\mathbf{I} \in \mathbf{M}$, and the notation for contractions

$$
A: B=\operatorname{tr}(A B)=\operatorname{tr}(B A)
$$

for $A, B \in \mathbf{M}$ will be used. For the euclidean matrix norm, we write $\|A\|=(A: A)^{1 / 2}$. Analytic functions such as square roots, logarithms 
and exponentials of matrices from $\mathbf{M}$ can be defined by the usual diagonalization construction. Note that $\operatorname{tr}(\log A)=0$ for all $A \in \mathbf{M}$. Positive constants whose values may change from line to line will be denoted by the same letter $K, L, \ldots$; constants that have a specific meaning and are referred to in other parts of the paper have indices.

2. Results for elongational deformations. The problem to be studied here has received much attention for flow histories of the form (7) with $b=c$, since it is then possible to find a solution of the same form for $t>0$. Here we give a brief overview of these results. In this case the system obtained from (5) and the incompressibility condition (6) reduces to only two equations for the two unknown functions $y(\cdot)=$ $a(\cdot)$ and $p(\cdot)$. One can then eliminate the pressure $p$ (see $\S 4$ for this procedure in the general case) and reduce the problem further to a single equation of the form

$$
0=\eta y^{\prime}(t)+\int_{-\infty}^{t} F(t-s, y(t), y(s)) d s
$$

for the elongation ratio $y$. For the separable case $F(s, \zeta, \xi)=$ $a(s) F_{0}(\zeta, \xi)$ this equation was studied in [11]. For a wide class of physically reasonable kernels $a(\cdot)$ and material functions $F_{0}(\cdot)$, the authors of this paper proved

(i) results on global existence, uniqueness, and comparison of solutions for the case $\eta>0$,

(ii) the existence of asymptotic limits in this case,

(iii) similar results for the implicit Volterra integrodifferential equation that results from setting $\eta=0$,

(iv) the existence of an initial layer for the solutions as $\eta \downarrow 0$, where the solution is governed by an ordinary differential equation on a fast time scale, and the uniform convergence of the solutions for large times, as $\eta \downarrow 0$,

(v) and some qualitative statements on the asymptotic behavior, including convergence rates and the result that for an important class of kernels and stretch histories the limit $y(\infty)$ of the elongation ratio will always be different from $y(-\infty)=1$.

In [12] and [14], (10) and another related scalar equation were studied numerically, and [12] contains also results for the case that a force is acting on the material sample for $t \geq 0$. In [7], a nonconvolution version of this equation was studied, and alternative proofs for some of the same results were given. In [1], a formal asymptotic expansion scheme for solutions of (10) (as $\eta \downarrow 0$ ) was described, and more precise estimates for the solutions were given in a special case. In [16] a 
refined model for filament stretching was discussed that incorporates inertial and boundary effects and leads to a scalar partial differential equation. For further results on elongational flows, we refer to [15].

3. Differential geometry of $\mathbf{M}$. In this section we equip the set $\mathbf{M}$, defined in (9), with the structure of a Riemannian manifold and derive the formulae for covariant differentiation and for geodesics on M. The reader is referred to [6] for background material. One easily verifies that $\mathbf{M}$ is a smooth five-dimensional submanifold of the linear space of symmetric $3 \times 3$-matrices; in fact, $\mathbf{M}$ is an unbounded closed strictly convex hypersurface, due to the concavity of the function $A \rightarrow$ $(\operatorname{det} A)^{1 / 3}$ on the cone of positive definite symmetric $3 \times 3$-matrices. For $A \in \mathbf{M}$, the tangent space at $A$ is given by

$$
T_{A} \mathbf{M}=\left\{B \in \mathbf{R}^{3,3} \mid B^{T}=B, B: A^{-1}=0\right\},
$$

where $A^{-1}=\operatorname{ad} A$ agrees with the adjoint of $A$ and therefore is a quadratic expression in the elements of $A$. Then the matrix exponential exp: $T_{\mathbf{I}} \mathbf{M} \rightarrow \mathbf{M}$ is a global diffeomorphism with inverse $\log : \mathbf{M} \rightarrow T_{\mathbf{I}} \mathbf{M}$.

Let $T \mathbf{M}$ be the tangent bundle of $\mathbf{M}$. Introduce a Riemannian structure on $T \mathbf{M}$ by defining the scalar products

$$
\langle B, C\rangle_{A}:=\operatorname{tr}\left(A^{-1} B A^{-1} C\right)=\left(A^{-1 / 2} B A^{-1 / 2}\right):\left(A^{-1 / 2} C A^{-1 / 2}\right)
$$

for $A \in \mathbf{M}$ and $B, C \in T_{A} \mathbf{M}$. Let $\|\cdot\|_{A}$ be the corresponding norm (and thus $\|\cdot\|_{\mathbf{I}}=\|\cdot\|$ ). The second formula shows that the mapping

$$
\Phi_{A}: T_{A} \mathbf{M} \rightarrow T_{\mathbf{I}} \mathbf{M}, \quad \Phi_{A}(B)=A^{-1 / 2} B A^{-1 / 2}
$$

is an isometry, and this suggests to define a covariant differentiation on $\mathbf{M}$ as follows: Let $J$ be an interval, $A: J \rightarrow \mathbf{M}$ be smooth, and $B: J \rightarrow T \mathbf{M}$ be a vectorfield along $A$, then set

$$
\begin{aligned}
\nabla_{A^{\prime}(t)} B(t) & =\Phi_{A(t)}^{-1}\left(\frac{d}{d t} \Phi_{A(t)} B(t)\right) \\
& =B^{\prime}(t)-\frac{1}{2}\left(A^{\prime}(t) A^{-1}(t) B(t)+B(t) A^{-1}(t) A^{\prime}(t)\right) .
\end{aligned}
$$

One checks that this is indeed the unique covariant differentiation (Levi-Civita connection) that is induced by the Riemannian structure. (It is sufficient to note that the product rule holds

$$
\frac{d}{d t}\langle X(t), X(t)\rangle_{A(t)}=2\left\langle\nabla_{A^{\prime}(t)} X(t), X(t)\right\rangle_{A(t)}
$$


and to compute that the torsion vanishes.) The fact that $\Phi_{A}$ is an isometry implies that parallel transport from $T_{A} \mathbf{M}$ to $T_{\tilde{A}} \mathbf{M}$ is given by $\Phi_{\tilde{A}}^{-1} \circ \Phi_{A}$ and thus path-independent, implying that also the curvature of this connection vanishes, i.e. that $\mathbf{M}$ is flat. Further, $\nabla_{A^{\prime}(t)} A^{\prime}(t)=A^{\prime \prime}(t)-A^{\prime}(t) A^{-1}(t) A^{\prime}(t)$, and geodesics in $\mathbf{M}$ are characterized by the second order equation $C^{\prime \prime}(t)=C^{\prime}(t) C^{-1}(t) C^{\prime}(t)$ and explicitly given by

$$
C(t)=A^{1 / 2} \exp \left(t \cdot \Phi_{A}(B)\right) A^{1 / 2} \quad\left(A \in \mathbf{M}, B \in T_{A} \mathbf{M}\right),
$$

where exp denotes the usual matrix exponential. The induced geodesic distance on $\mathbf{M}$ can thus be computed as

$$
d(A, B)=\left\|\log \left(A^{-1 / 2} B A^{-1 / 2}\right)\right\|=\left\|\log \left(B^{-1 / 2} A B^{-1 / 2}\right)\right\| .
$$

Finally we note that the function $(A, B) \rightarrow A: B^{-1}$ on $\mathbf{M} \times \mathbf{M}$ has the properties

$$
A: B^{-1} \geq 3
$$

with equality if and only if $A=B$,

$$
\lambda \cdot\|A-B\|^{2} \leq A: B^{-1}-3 \leq \Lambda \cdot\|A-B\|^{2}
$$

for any $A, B \in \mathbf{K} \subset \mathbf{M}$ compact, with $\lambda, \Lambda$ depending on $\mathbf{K}$, and

$$
A: B^{-1} \rightarrow \infty \Leftrightarrow\left\|A B^{-1}\right\| \rightarrow \infty \text {. }
$$

The proofs of these statements are left to the reader.

4. Gradient structure and local existence. Returning to problem (5), we show in this section that the problem has a natural "gradient structure", and we give conditions for a basic local existence and uniqueness result, if $\eta>0$. To simplify the notation, we shall write from now on $C(t)=\gamma^{-1}(t)$. Then (5) together with (4) takes the form

$$
\begin{aligned}
p(t) C(t)+\eta C^{\prime}(t) & \\
= & \int_{-\infty}^{t}\left(W_{1}\left(t-s, \lambda_{1}(t, s), \lambda_{2}(t, s)\right) C(s)\right. \\
& \left.-W_{2}\left(t-s, \lambda_{1}(t, s), \lambda_{2}(t, s)\right) C(t) C^{-1}(s) C(t)\right) d t
\end{aligned}
$$

on $\mathbf{M}$, where $W_{1}, W_{2}, \lambda_{1}, \lambda_{2}$ have the same meaning as above. Equations (16) and (17) below will be referred to as $\left(16_{\eta}\right),\left(17_{\eta}\right)$ resp. as $\left(16_{0}\right),\left(17_{0}\right)$, depending on whether $\eta>0$ or $\eta=0$ in (5). Similarly, we shall refer to solutions of these equations as $C^{\eta}(\cdot)$ resp. as $C^{0}(\cdot)$. Let $F: \mathbf{M} \times \mathbf{M} \rightarrow \mathbf{R}$ be a smooth function of the form

$$
F(A, B)=\psi\left(A^{-1}: B, A: B^{-1}\right) \text {, }
$$


and define as usual the gradient of $F$ with respect to its first argument as the element of $T_{A} \mathbf{M}$ satisfying

$$
\left\langle\operatorname{grad}_{A} F(A, B), C\right\rangle_{A}=\left.\frac{d}{d t} F(\tilde{A}(t), B)\right|_{t=0}
$$

for all $C \in T_{A} \mathbf{M}$, where $A$ is continued as a curve in $\mathbf{M}$ with $\tilde{A}(0)=$ $A, \widetilde{A}^{\prime}(0)=C$. A simple calculation then shows that

$$
\operatorname{grad}_{A} F(A, B)=\lambda A-\psi_{1} \cdot B+\psi_{2} \cdot A B^{-1} A
$$

with $\lambda \in \mathbf{R}$ given by

$$
\lambda=\frac{1}{3}\left(\psi_{1} \cdot A^{-1}: B-\psi_{2} \cdot A: B^{-1}\right) .
$$

Here, $\psi_{i}=\left(\partial / \partial \lambda_{i}\right) \psi\left(\lambda_{1}, \lambda_{2}\right)$. This argument shows that $\left(16_{\eta}\right)$ can also be written in the form

$$
\begin{aligned}
\eta C^{\prime}(t) & =-\int_{-\infty}^{t} \operatorname{grad}_{A} H(t-s, C(t), C(s)) d s \\
& =-\int_{-\infty}^{t} h(t-s, C(t), C(s)) d s
\end{aligned}
$$

as an equation that holds in $T_{C(t)} \mathbf{M}$, with

$$
\begin{aligned}
H(s, A, B) & =W\left(s, A^{-1}: B, A: B^{-1}\right), \\
h(s, A, B) & =\operatorname{grad}_{A} H(s, A, B) \in T_{A} \mathbf{M} .
\end{aligned}
$$

Here and below $\operatorname{grad}_{A} H$ denotes the gradient of $H$ with respect to its second argument. This "abstract" form of $\left(16_{\eta}\right)$ shows that the equation has a gradient structure, if the Riemannian metric on TM is chosen as above.

Moreover, this observation shows how to eliminate the pressure $p(\cdot)$ from $\left(16_{\eta}\right)$ : Taking the contraction with $C^{-1}(t)$ and noting that $C^{\prime}(t): C^{-1}(t)=0$, we obtain

$$
\begin{aligned}
3 p(t)=\int_{-\infty}^{t}\left(W_{1}(t-s,\right. & \left.\lambda_{1}(t, s), \lambda_{2}(t, s)\right) \lambda_{1}(t, s) \\
& \left.-W_{2}\left(t-s, \lambda_{1}(t, s), \lambda_{2}(t, s)\right) \lambda_{2}(t, s)\right) d s .
\end{aligned}
$$

Inserting this back into $\left(16_{\eta}\right)$, we obtain an equation for $C$ alone; for the case $\eta>0$, it is easy to see that $\operatorname{det} C(t)$ remains constant along any solution of this equation. We next list a set of basic assumptions which will always be used in the sequel. 
(H0) The partial derivatives

$$
\begin{gathered}
W^{\prime}=\frac{\partial}{\partial s} W, \quad W_{i}=\frac{\partial}{\partial \lambda_{i}} W, \quad W_{i}^{\prime}=\frac{\partial^{2}}{\partial s \partial \lambda_{i}} W \quad \text { and } \\
W_{i j}=\frac{\partial^{2}}{\partial \lambda_{i} \partial \lambda_{j}} W
\end{gathered}
$$

are measurable and bounded; for any $R>0$, there exists $m_{R} \in$ $L^{1}(0, \infty ; \mathbf{R})$ such that for all $s>0,3 \leq \lambda_{i} \leq R$ and all $i, j$

$$
\begin{aligned}
\left|W_{i}\left(s, \lambda_{1}, \lambda_{2}\right)\right|,\left|W_{i j}\left(s, \lambda_{1}, \lambda_{2}\right)\right| & \leq m_{R}(s), \\
\left|W^{\prime}\left(s, \lambda_{1}, \lambda_{2}\right)\right|,\left|W_{i}^{\prime}\left(s, \lambda_{1}, \lambda_{2}\right)\right| & \leq m_{R}(s) .
\end{aligned}
$$

The function $C_{0}:(-\infty, 0) \rightarrow \mathbf{M}$ is measurable and bounded, and $C_{00} \in \mathbf{M}$ is a given matrix. For all $s>0, W(s, 3,3)=0$.

Under these assumptions the (covariant) derivative

$$
\nabla \operatorname{grad}_{A} H(s, A, B)=\nabla h(s, A, B)
$$

(with respect to the second argument) exists as a linear self-map of $T_{A} \mathbf{M}$ for all $s, A, B$ and satisfies an estimate of the form

$$
\left\|\nabla \operatorname{grad}_{A} H(s, A, B)\right\| \leq m_{R}(s),
$$

with $R=\|A\|+\|B\|$. As usual, we shall write $\nabla_{C} \operatorname{grad}_{H}(s, A, B)$ for the image of an element $C \in T_{A} \mathbf{M}$ under this map. We shall compute this derivative in $\S 6$ below.

Local existence and uniqueness of solutions for $\left(16_{\eta}\right)$ now follow by eliminating the pressure as shown above, converting the integrodifferential equation into an integral equation, and applying a standard contraction argument (see e.g. [13]).

Proposition 4.1. Let $\eta>0$. Then there exists a unique solution $C^{\eta}(\cdot)$ of $\left(16_{\eta}\right)$ on some maximal interval $\left[0, t_{0}\right)$ that satisfies $C^{\eta}(0)=$ $C_{00}$ and is continued as $C_{0}(\cdot)$ on $(-\infty, 0)$. If $t_{0}<\infty$, then

$$
\limsup _{t \rightarrow t_{0}}\left\|C^{\eta}(t)\right\|=\infty
$$

For the proof of this proposition the gradient structure (17) need not be assumed; also, assumption (19) in (H0) is not necessary.

5. Global properties for $\eta>0$. According to the previous proposition, it suffices to give an a priori estimate for a solution of $\left(16_{\eta}\right)$ to guarantee its global existence. Such an estimate will be derived in this section by an energy argument that will also allow us to deduce the 
existence of an asymptotic limit, using the gradient structure $\left(17_{\eta}\right)$. We assume throughout this section that $\eta>0$ and write $C(\cdot)$ for $C^{\eta}(\cdot)$. The following additional hypotheses will be used:

(H1) For some $K_{0} \geq 0, m(\cdot) \in L^{1}(0, \infty ; \mathbf{R})$ and for all $\left(s, \lambda_{1}, \lambda_{2}\right)$,

$$
\begin{gathered}
W\left(s, \lambda_{1}, \lambda_{2}\right)+m(s) \geq 0 \\
W^{\prime}\left(s, \lambda_{1}, \lambda_{2}\right) \leq K_{0}\left(W\left(s, \lambda_{1}, \lambda_{2}\right)+m(s)\right) .
\end{gathered}
$$

(H2) There exists some $\delta>0$ such that for all $\left(s, \lambda_{1}, \lambda_{2}\right)$

$$
\begin{gathered}
W\left(s, \lambda_{1}, \lambda_{2}\right) \geq 0, \\
W^{\prime}\left(s, \lambda_{1}, \lambda_{2}\right)+\delta W\left(s, \lambda_{1}, \lambda_{2}\right) \leq 0, \\
\inf _{t \geq 0} e^{\delta t} \int_{t}^{\infty} W\left(s, \lambda_{1}, \lambda_{2}\right) d s \rightarrow \infty \quad \text { as } \lambda_{1}, \lambda_{2} \rightarrow \infty .
\end{gathered}
$$

To illustrate these assumptions, consider the separable case

$$
W\left(s, \lambda_{1}, \lambda_{2}\right)=a(s) W_{0}\left(\lambda_{1}, \lambda_{2}\right)
$$

with $a(\cdot) \in L^{1}(0, \infty ; \mathbf{R})$. Then (H0) holds if $a$ is locally absolutely continuous with $\left|a^{\prime}(\cdot)\right| \in L^{1}(0, \infty ; \mathbf{R})$ and if $W_{0}$ is sufficiently smooth; (2) holds if $W_{0}$ is bounded from below, and (21) is true if additionally $a^{\prime} \leq K_{0} a$ on $(0, \infty)$. Further, (22) holds if $W_{0}$ and $a$ are non-negative, (23) follows if additionally $a^{\prime}+\delta a \leq 0$, and (24) is implied by the assumption that $W_{0}\left(\lambda_{1}, \lambda_{2}\right) \rightarrow \infty$ as $\lambda_{1}, \lambda_{2} \rightarrow \infty$ and

$$
\int_{t}^{\infty} a(s) d s \geq c e^{-\delta t}
$$

for some $c>0$, for all $t \geq 0$. In particular, (23) and (24) certainly follow if $a$ is the Laplace-Stieltjes transform of a non-negative finite Borel-measure $\mu$ with support on $[\delta, \infty)$ such that $\mu(\{\delta\})>0$. Such kernels occur frequently in applications, where the support of $\mu$ is a discrete set of reciprocals of relaxation times (see [9] and [15] for more details).

THEOREM 5.1. Assume that (H0) holds.

(a) If (H1) holds, then the unique solution of $\left(16_{\eta}\right)$ exists for all $t>0$.

(b) If (H2) (22) and (23) hold, then there exist $C_{\infty} \in \mathbf{M}$ and $K=$ $K\left(\eta, C_{0}(\cdot), C_{00}\right)>0$ such that

$$
\left\|C(t)-C_{\infty}\right\| \leq K \cdot e^{-\delta t / 2}
$$


(c) If (H2) (24) holds, then for some $K=K\left(\sup _{t<0}\left\|C_{0}(t)\right\|, C_{00}\right)$ that is independent of $\eta$

$$
\sup _{t \geq 0}\|C(t)\| \leq K
$$

To prove these results, we use a suitable energy identity.

LEMMA 5.2. Let $H:(0, \infty) \times \mathbf{M} \times \mathbf{M} \rightarrow \mathbf{R}$ be defined as in $\S 4$, and set $H^{\prime}(s, A, B)=d / d s H(s, A, B)$. Let $C(\cdot)$ be any solution of $\left(16_{\eta}\right)$ on $\left(0, t_{0}\right)$, then for all $t \in\left(0, t_{0}\right)$

$$
\begin{aligned}
\eta \cdot\left\|C^{\prime}(t)\right\|_{C(t)}^{2}+\frac{d}{d t}\left(\int_{-\infty}^{t} H(t-s, C(t), C(s)) d s\right) \\
=\int_{-\infty}^{t} H^{\prime}(t-s, C(t), C(s)) d s .
\end{aligned}
$$

Proof of Lemma 5.2. The identity follows by differentiating

$$
\int_{-\infty}^{t} H(t-s, C(t), C(s)) d s
$$

with respect to $t$ and using $\left(17_{\eta}\right)$ and the definition of the Riemannian structure on $T \mathbf{M}$.

Proof of Theorem 5.1. Using (21) in (28) and integrating from 0 to $t<t_{0}$, where $t_{0}$ is the length of the maximal existence interval, implies the identity

$$
\begin{aligned}
& \eta \cdot \int_{0}^{t}\left\|C^{\prime}(s)\right\|_{C(s)}^{2} d s+\int_{-\infty}^{t} H(t-s, C(t), C(s)) d s \\
& \quad \leq K\left(1+\int_{0}^{t} \int_{-\infty}^{s} H(s-\tau, C(s), C(\tau)) d \tau d s\right) .
\end{aligned}
$$

Since $H$ is uniformly bounded from below, this implies that

$$
\int_{0}^{t}\left\|C^{\prime}(s)\right\|_{C(s)}^{2} d s
$$

remains bounded as $t \uparrow t_{0}$. Therefore the solution can be continued past $t_{0}$, unless $t_{0}=\infty$. Part (a) is proved.

To prove parts (b) and (c), we multiply (28) with $e^{\delta t}$ and note that the result can be written as

$$
\begin{array}{r}
e^{\delta t} \eta\left\|C^{\prime}(t)\right\|_{C(t)}^{2}+\frac{d}{d t}\left(e^{\delta t} \int_{-\infty}^{t} H(t-s, C(t), C(s)) d s\right) \\
=e^{\delta t} \int_{-\infty}^{t}\left(H^{\prime}(t-s, C(t), C(s))\right. \\
+\delta H(t-s, C(t), C(s))) d s .
\end{array}
$$


Here the right hand side is nonpositive by (H1) (23). Integrating this estimate gives

$$
\eta \int_{0}^{t} e^{\delta s}\left\|C^{\prime}(s)\right\|_{C(s)}^{2} d s+e^{\delta t} \int_{-\infty}^{t} H(t-s, C(t), C(s)) d s \leq L
$$

where $L$ depends only on $\sup _{t \leq 0}\left\|C_{0}(t)\right\|$ and on $\left\|C_{00}\right\|$. Dropping the second term and recalling the definition of the scalar product on the left hand side implies that

$$
t \rightarrow \sqrt{\eta} e^{\delta t / 2} \cdot C^{\prime}(t) \cdot C^{-1}(t)
$$

is uniformly bounded in $L^{2}\left(0, \infty ; \mathbf{R}^{3,3}\right)$. A standard Gronwall argument implies first that

$$
\|C(t)\|=O\left(\exp \left(\frac{k}{\sqrt{\eta \delta}}\right)\right)
$$

for some $K>0$ uniformly in $t$. Thus (31) implies also that

$$
\|C(t)-C(s)\|=O\left(\exp \left(\frac{K}{\sqrt{\eta \delta}}\right)\right) \cdot \frac{e^{-\delta t / 2}}{\sqrt{\eta \delta}}
$$

for all $0<t<s$, and (26) follows.

To prove part (c), we drop the first term in (30) and derive the estimate

$$
e^{\delta t} \int_{-\infty}^{0} W\left(t-s, C^{-1}(t): C_{0}(s), C(t): C_{0}^{-1}(s)\right) d s \leq L
$$

for all $t$. By (H2) (24), $C^{-1}(t): C_{0}(s)+C(t): C_{0}^{-1}(s)$ cannot exceed a certain bound, if $s$ varies in a suitable subset of $(-\infty, 0)$ with positive measure. Then a uniform bound for the $C(t)$ follows from (15) and the essential boundedness of $C_{0}(\cdot)$ on $(-\infty, 0)$.

The $L^{2}$-estimate (30) can be viewed as a bound on the "logarithmic derivative" of $C(\cdot)$. In [11], pointwise estimates of the derivative of $C(\cdot)$ itself (in the scalar version (10)) were obtained by means of comparison arguments.

6. Solutions for $\eta=0$. If the viscosity $\eta$ vanishes, then the reduced equation $\left(16_{0}\right)$ and its abstract version $\left(17_{0}\right)$ are implicit Volterra in. tegral equations for $C(t)$, and in general it is not possible to isolate $C(t)$ from them (cf. [1] for some exceptions in the case of elongational flows). In this section we use the gradient structure of $\left(17_{0}\right)$ to identify conditions under which local and global existence and uniqueness 
results hold, and we also deduce the existence of asymptotic limits of solutions as $t \rightarrow \infty$.

From $\left(17_{0}\right)$ it follows that $C(\cdot)$ is a solution on $\left[0, t_{0}\right)$ if and only if for any $0 \leq t<t_{0}$ the matrix $C(t)$ is a stationary point of the functional defined by

$$
\begin{aligned}
A & \rightarrow \int_{0}^{\infty} W\left(s, A^{-1}: C(t-s), A: C^{-1}(t-s)\right) d s \\
& =\int_{0}^{\infty} H(s, A, C(t-s)) d s \quad(A \in \mathbf{M}) .
\end{aligned}
$$

Some important properties of functionals of this type can be deduced from the following lemma.

LeMMA 6.1. Let $\mathbf{F}: \mathbf{M} \times \mathbf{M} \rightarrow \mathbf{R}$ be given by

$$
\mathbf{F}(A, B)=\psi\left(A^{-1}: B, A: B^{-1}\right),
$$

where $\psi$ is a smooth function.

(a) Then for any $C \in T_{A} \mathbf{M}$ the covariant derivative of $\operatorname{grad}_{A} \mathbf{F}$ with respect to its argument satisfies

$$
\begin{aligned}
& \left\langle\nabla_{C} \operatorname{grad}_{A} \mathbf{F}(A, B), C\right\rangle_{A} \\
& \quad=\psi_{1} \cdot A^{-1} C A^{-1} C A^{-1}: B+\psi_{2} \cdot C A^{-1} C: B^{-1} \\
& \quad+\psi_{11} \cdot\left(A^{-1} C A^{-1}: B\right)^{2}+\psi_{22} \cdot\left(C: B^{-1}\right)^{2} \\
& \quad-2 \psi_{12} \cdot\left(A^{-1} C A^{-1}: B\right)\left(C: B^{-1}\right) .
\end{aligned}
$$

Here $\psi_{i}$ and $\psi_{i j}$ are the partial derivatives of $\psi$, and their arguments are $A^{-1}: B$ and $A: B^{-1}$.

(b) If the function

$$
(\xi, \zeta) \rightarrow \psi\left(e^{\xi}, e^{\zeta}\right)
$$

is convex for $\xi, \zeta \geq \log 3$, and if $\psi_{1}(3,3), \psi_{2}(3,3) \geq 0$, then for any $C \in T_{A} \mathbf{M}$

$$
\begin{aligned}
& \left\langle\nabla_{C} \operatorname{grad}_{A} \mathbf{F}(A, B), C\right\rangle_{A} \\
& \quad \geq\left(\left(A^{-1}: B\right)^{-1} \psi_{1}+\left(A: B^{-1}\right)^{-1} \psi_{2}\right)\|C\|_{A}^{2} .
\end{aligned}
$$

In particular, if $\psi_{1}(3,3)+\psi_{2}(3,3)>0$, then the right hand side of (35) is bounded below by $c\|C\|_{A}^{2}$ with $c=c(A, B)>0$.

Proof. To prove part (a), let $A(t)=A^{1 / 2} \exp \left(t \Phi_{A}(C)\right) A^{1 / 2}$ be the geodesic through $A$ with initial velocity $A^{\prime}(0)=C$. Then

$$
\left.\frac{d^{2}}{d t^{2}} F(A(t), B)\right|_{t=0}=\left\langle\nabla_{C} \operatorname{grad}_{A} F(A, B), C\right\rangle_{A},
$$


since $\nabla_{A^{\prime}(t)} A^{\prime}(t) \equiv 0$. A direct computation of the left hand side of this identity then proves (33).

For the proof of part (b), we set $D=A^{-1 / 2} B A^{-1 / 2}$ and $C^{*}=$ $\Phi_{A}(C)$. The left hand side of (33) can then be written as

$$
\begin{aligned}
\psi_{11}\left(C^{*}: D\right)^{2} & -2 \psi_{12}\left(C^{*}: D\right)\left(C^{*}: D^{-1}\right)+\psi_{22}\left(C^{*}: D^{-1}\right)^{2} \\
& +\psi_{1} \operatorname{tr}\left(C^{*} D C^{*}\right)+\psi_{2} \operatorname{tr}\left(C^{*} D^{-1} C^{*}\right)
\end{aligned}
$$

Now

$$
\left(C^{*}: D\right)^{2}=\left(\operatorname{tr}\left(\left(D^{1 / 2} C^{*}\right)^{T} D^{1 / 2}\right)\right)^{2} \leq \operatorname{tr}\left(C^{*} D C^{*}\right) \cdot \operatorname{tr} D
$$

by Cauchy-Schwarz. Since $\operatorname{tr} C^{*}=0$, we can replace $D$ with the positive definite matrix $D_{1}=D-\varepsilon \operatorname{tr} D \mathbf{I}, \varepsilon=\left(\operatorname{tr} D \operatorname{tr} D^{-1}\right)^{-1}=$ $\left(\left(A^{-1}: B\right)\left(A: B^{-1}\right)\right)^{-1}$, without changing the left hand side. The right hand side now becomes

$$
(1-3 \varepsilon) \operatorname{tr} D\left(\operatorname{tr}\left(C^{*} D C^{*}\right)-\varepsilon \operatorname{tr} D\left\|C^{*}\right\|^{2}\right) .
$$

Rearranging the resulting inequality implies

$$
\operatorname{tr}\left(C^{*} D C^{*}\right) \geq\left(\operatorname{tr} D^{-1}\right)^{-1}\left\|C^{*}\right\|^{2}+(\operatorname{tr} D)^{-1}\left(C^{*}: D\right)^{2} .
$$

A similar estimate holds if $D$ is replaced with $D^{-1}$. Thus the expression in (36) is bounded below by

$$
\begin{aligned}
\left(\psi_{11}\right. & \left.+\psi_{1}(\operatorname{tr} D)^{-1}\right)\left(C^{*}: D\right)^{2}-2 \psi_{12}\left(C^{*}: D\right)\left(C^{*}: D^{-1}\right) \\
& +\left(\psi_{22}+\psi_{2}\left(\operatorname{tr} D^{-1}\right)^{-1}\right)\left(C^{*}: D^{-1}\right)^{2} \\
& +\left(A: B^{-1} \psi_{1}+A^{-1}: B \psi_{2}\right)\left\|C^{*}\right\|^{2} .
\end{aligned}
$$

Recall now that the arguments of $\psi$ are $\operatorname{tr} D=A^{-1}: B$ and $\operatorname{tr} D^{-1}=$ $A: B^{-1}$. One then checks easily that the quadratic form in $\left(C^{*}: D\right.$, $\left.C^{*}: D^{-1}\right)$ in this expression is positive semidefinite if and only if (34) holds. In particular, if $\psi_{1}(3,3)+\psi_{2}(3,3)>0$, then $\psi_{1}\left(\lambda_{1}, \lambda_{2}\right)+$ $\psi_{2}\left(\lambda_{1}, \lambda_{2}\right) \geq \delta_{R}>0$ whenever $3 \leq \lambda_{1}, \lambda_{2} \leq R$, and the last assertion of part (b) follows.

The reduced problem $\left(16_{0}\right)$ will be (locally) uniquely solvable if some of the assumptions in the above Lemma hold for the functionals appearing in (32). Set $\rho(s)=W_{1}(s, 3,3)+W_{2}(s, 3,3)$, then the key hypothesis is

(H3) $(\xi, \zeta) \rightarrow W\left(s, e^{\xi}, e^{\zeta}\right)$ is convex on $[3, \infty) \times[3, \infty)$ for all $s>0, W_{i}(s, 3,3) \geq 0(i=1,2 ; s>0), \int_{0}^{\infty} \rho(s) d s>0$. 
LemMA 6.2. Let $W$ satisfy $(\mathrm{H} 0)$ and (H3). Let $B \in L^{\infty}(-\infty, 0 ; \mathbf{M})$ and define the functional

$$
\begin{aligned}
\mathbf{G}(A ; B(\cdot)) & =\int_{0}^{\infty} H(s, A, B(-s)) d s \\
& =\int_{-\infty}^{0} W\left(-s, A^{-1}: B(s), A: B^{-1}(s)\right) d s
\end{aligned}
$$

for $A \in \mathbf{M}$.

(a) Then for any $A \in \mathbf{M}$ there exists $\varepsilon=\varepsilon\left(A,\|B(\cdot)\|_{\infty}\right)>0$ such that for all $C \in T_{A} \mathbf{M}$

$$
\left\langle\nabla_{C} \operatorname{grad}_{A} \mathbf{G}(A, B(\cdot)), C\right\rangle_{A} \geq \varepsilon\|C\|_{A}^{2} .
$$

(b) There exists exactly one stationary point $A$ of $\mathbf{G}(\cdot, B(\cdot))$ on $\mathbf{M}$, and the mapping $B(\cdot) \rightarrow A$ is bounded on bounded sets of $L^{\infty}(0, \infty ; \mathbf{M})$.

(c) Let $R>0$, then there exists $m_{R} \in L^{1}(-\infty, 0 ; \mathbf{R})$ such that for any $B_{1}, B_{2} \in L^{\infty}(-\infty, 0 ; \mathbf{M})$ with $\left\|B_{i}(\cdot)\right\|_{\infty} \leq R$ the stationary points $A_{i}$ of $\mathbf{G}\left(\cdot, B_{i}(\cdot)\right)$ satisfy

$$
\left\|A_{1}-A_{2}\right\| \leq \int_{-\infty}^{0} m_{R}(s)\left\|B_{1}(s)-B_{2}(s)\right\| d s .
$$

Proof. Part (a) follows directly from (H3) by integrating and using Lemma 6.1(b). To prove part (b), we first note that (H3) also implies that

$$
W\left(s, \lambda_{1}, \lambda_{2}\right) \geq W_{1}(s, 3,3) \log \frac{\lambda_{1}}{3}+W_{2}(s, 3,3) \log \frac{\lambda_{2}}{3}
$$

and therefore $\mathbf{G}(A, B(\cdot)) \rightarrow \infty$ as $\|A\| \rightarrow \infty$, uniformly for $B(\cdot)$ in bounded sets in $L^{\infty}(-\infty, 0 ; \mathbf{M})$. Thus $\mathbf{G}(\cdot, B(\cdot))$ has at least one stationary point (a global minimum). However, since (a) is equivalent to the strict convexity of any function $t \rightarrow \mathbf{G}(A(t), B(\cdot))$, if $t \rightarrow A(t)$ is any geodesic in $\mathbf{M}$, no other stationary points can exist, proving (b).

To prove part (c), let $B_{1}, B_{2} \in L^{\infty}(0, \infty ; \mathbf{M}), A \in \mathbf{M}$ and $C \in$ $T_{A} \mathbf{M}$. Then by a direct estimation, using ( $\left.\mathrm{H} 0\right)$

$$
\begin{gathered}
\left\|\nabla_{C}\left(\operatorname{grad}_{A} \mathbf{G}\left(A, B_{1}(\cdot)\right)-\operatorname{grad}_{A} \mathbf{G}\left(A, B_{2}(\cdot)\right)\right)\right\|_{A} \\
\quad \leq \int_{-\infty}^{0} k_{R}(s)\left\|B_{1}(s)-B_{2}(s)\right\| d s
\end{gathered}
$$

for a suitable $k_{R} \in L^{1}(-\infty, 0 ; \mathbf{R}), R \geq\left\|B_{i}(\cdot)\right\|_{\infty}$. Moreover, the map

$$
C \rightarrow \nabla_{C} \operatorname{grad}_{A} \mathbf{G}(A, B(\cdot))
$$


mapping $T_{A} \mathbf{M}$ into itself is nonsingular by part (a). By the implicit function theorem and standard results on the dependence of parameters (see [18]), the assertion follows.

A basic existence result for solutions of the reduced equation $\left(16_{0}\right)$ can now be deduced.

THeOREM 6.3. Let $W$ satisfy hypotheses (H0) and (H3). Then there exist $t_{0}>0$ and a unique continuously differentiable function $C^{0}:\left[0, t_{0}\right) \rightarrow \mathbf{M}$ such that $\left(16_{0}\right)$ holds for all $0 \leq t<t_{0}$, with $C^{0}$ continued as $C_{0}$ on $(-\infty, 0)$. Moreover, if the maximal existence interval $\left[0, t^{*}\right)$ of the solution is bounded, then $\left\|C^{0}(t)\right\| \rightarrow \infty$ as $t \uparrow t^{*}$.

Proof. The proof uses a standard contraction mapping argument. By Lemma 6.2, there exists exactly one stationary point $C^{00}$ of the function $\mathbf{G}\left(\cdot, C_{0}(\cdot)\right)$. For $R, t>0$, let $\mathbf{X}_{R, t}$ be the complete metric space

$\mathbf{X}_{R, t}=\left\{B \in C([0, t], \mathbf{M}) \mid B(0)=C^{00},\left\|B(s)-C^{00}\right\| \leq R(0 \leq s \leq t)\right\}$

equipped with the supremum metric $d(\cdot, \cdot)$. For any element $B \in$ $\mathbf{X}_{R, t}, 0 \leq s \leq t,-\infty<\tau<0$, set $B_{s}(\tau)=C_{0}(s+\tau)$, if $s+\tau<$ $0, B_{s}(\tau)=B(s+\tau)$, if $0 \leq s+\tau \leq t$, and define $\mathbf{F}(B(\cdot)):=A(\cdot)$ by requiring that $A(s) \in \mathbf{M}$ is the stationary point of $\mathbf{G}\left(\cdot, B_{s}(\cdot)\right)$. Then $\mathbf{F}: \mathbf{X}_{R, t} \rightarrow L^{\infty}(0, t ; \mathbf{M})$ is well-defined.

We next note that images of $\mathbf{F}$ are continuous functions: Let $A\left(s_{i}\right)$ $=\mathbf{F}(B(\cdot))\left(s_{i}\right) \quad(i=0,1)$. Then by Lemma $6.2(\mathrm{c})$

$$
\left\|A\left(s_{0}\right)-A\left(s_{1}\right)\right\| \leq \int_{-\infty}^{0} m_{R}(-\tau)\left\|B_{s_{0}}(\tau)-B_{s_{1}}(\tau)\right\| d \tau
$$

with some fixed $m_{R} \in L^{1}(0, \infty ; \mathbf{R})$. The right hand side of this inequality will go to zero as $\left|s_{0}-s_{1}\right| \rightarrow 0$, as a standard argument shows (approximate $m_{R}$ by a bounded function with compact support and use Egorov's theorem). This estimate, with $s_{0}=0, A\left(s_{0}\right)=C^{00}$, also shows that $\mathbf{F}$ will map $\mathbf{X}_{R, t}$ into itself, if $t$ is sufficiently small. Finally, if $B_{i} \in \mathbf{X}_{R, t}$ and $A_{i}=\mathbf{F}\left(B_{i}\right)$ for $i=0,1$, then by Lemma 6.2(c)

$$
d\left(A_{0}, A_{1}\right) \leq \int_{0}^{t} m_{R}(s) d s \cdot d\left(B_{0}, B_{1}\right)
$$

and $\mathbf{F}$ will be a contraction, if $t$ is reduced further. By Banach's Fixed Point Theorem, this implies the existence and uniqueness of a local continuous solution $C^{0}(\cdot)$. 
We next note that solutions can only cease to exist if they become unbounded: If $\left\|C^{0}(s)\right\|$ is bounded on some interval $\left[0, t_{0}\right]$, then $C^{0}$ must have a limit as $t \uparrow t_{0}$, by the same argument that proved the continuity of images of $\mathbf{F}$ above. Then the solution can be continued past $t_{0}$.

Finally, the continuous differentiability of the solution $C^{0}(\cdot)$ for $t \geq 0$ follows by noting that $C^{0}(t)$ is the unique point on $\mathbf{M}$ at which the vector field

$$
A \rightarrow \int_{-\infty}^{t} \operatorname{grad}_{A} H\left(t-s, A, C^{0}(s)\right) d s \in T_{A} \mathbf{M}
$$

has a zero. Here $C^{0}(s)=C_{0}(s)$ for $s<0$. If $C^{0}$ is continuous for $t \geq 0$, this family of vector fields on $T \mathbf{M}$ is continuously differentiable in $t$ by (H0). The implicit function theorem implies that $C^{0}$ is continuously differentiable.

The final result of this section gives global existence of solutions and the existence of asymptotic limits under essentially the same conditions as in the case $\eta>0$. We drop the superscript and write $C$ for $C^{0}$ in the rest of this section.

THEOREM 6.4. Let $W$ satisfy hypotheses $(\mathrm{H} 0),(\mathrm{H} 2)$ with some $\delta>$ 0 , and (H3). Then there exist a unique $C^{1}$-solution $C(\cdot)$ of $\left(16_{0}\right)$ on $[0, \infty)$, some $C_{\infty} \in \mathbf{M}$ and some $\varepsilon>0$ such that $\left\|C(t)-C_{\infty}\right\|=$ $O\left(e^{-\varepsilon t}\right)$ as $t \rightarrow \infty$.

Proof. Let $C(\cdot)$ be the unique local solution of $\left(16_{0}\right)$ with maximal existence interval $\left[0, t^{*}\right)$. Define the energy

$$
\begin{aligned}
E(t) & =\int_{0}^{\infty} H(s, C(t), C(t-s)) d s \\
& =\int_{-\infty}^{t} W\left(t-s, C^{-1}(t): C(s), C(t): C^{-1}(s)\right) d s .
\end{aligned}
$$

We claim that for all $t \in\left[0, t^{*}\right)$

$$
E(t) \leq e^{-\delta t} E(0)
$$

where $\delta$ is as in (H2). This estimate can be proved in the same way as Theorem 5.1(b) and (c), i.e. by showing the energy identity (28) and deriving (30) from it, since $C(\cdot)$ is differentiable. We want to give a slightly different argument for (43) that does not require the solution 
to be differentiable. Let $0 \leq t<t+\tau<t^{*}$. Since $C(t+\tau)$ minimizes a functional of the form (38), we have

(44) $e^{\delta(t+\tau)} E(t+\tau)$

$$
\leq e^{\delta \tau} \int_{-\infty}^{t+\tau} W\left(t+\tau-s, C^{-1}(t): C(s), C(t): C^{-1}(s)\right) d s .
$$

Now (H2) implies that $W\left(\sigma+\tau, \lambda_{1}, \lambda_{2}\right) \leq e^{-\delta \tau} W\left(\sigma, \lambda_{1}, \lambda_{2}\right)$ for all $\sigma \geq 0, \lambda_{1}, \lambda_{2} \geq 3$. Applying this to the part of the integral in (44) that extends from $-\infty$ to $t$ and noting that the remaining integral

$$
e^{\delta \tau} \int_{0}^{\tau} W\left(s, C^{-1}(t): C(t+\tau-s), C(t): C^{-1}(t+\tau-s)\right) d s
$$

is uniformly of the order $O(\tau)$ and in fact $o(\tau)$ if $t$ is a Lebesgue point of $C(\cdot)$, we deduce (43). From (43), a global uniform a priori bound for the solution follows just as in the proof of Theorem 5.1(c). Therefore, the solution exists for all times and is uniformly bounded. Moreover, for some small $\sigma>0$, sufficiently large $K>0$, and a suitable $s_{1}>\sigma$, by (41) and (43)

$$
\begin{aligned}
\int_{t-\sigma}^{t} & \|C(t)-C(s)\| d s \leq K \int_{t-\sigma}^{t}-\left(W_{1}\left(s_{1}, 3,3\right) \log \frac{C^{-1}(t): C(s)}{3}\right. \\
& \left.\quad+W_{2}\left(s_{1}, 3,3\right) \log \frac{C(t): C^{-1}(s)}{3}\right) d s \\
& \leq K \int_{t-\sigma}^{t} W\left(s_{1}, C^{-1}(t): C(s), C(t): C^{-1}(s)\right) d s \\
& \leq K E(t) \leq K e^{-\delta t} E(0) .
\end{aligned}
$$

Thus,

$$
C(t)-\sigma^{-1} \int_{t-\sigma}^{t} C(s) d s=O\left(e^{-\delta t}\right)
$$

Then well-known results on linear Volterra equations (see [13]) imply that $C(\cdot)$ must tend to some limit $C_{\infty}$ at an exponential rate.

7. The initial layer. In this section, we investigate the behavior of solutions of $\left(16_{\eta}\right)$ as $\eta$ tends to 0 , for small $t$. It is shown that the solution behavior is approximately governed by an ordinary differential equation on a fast time scale, whose solutions tend to the limit $C^{00}=C^{0}(0)$, the natural initial value of the solution of the reduced equation $\left(16_{0}\right)$. These are natural extensions of the results given in [11]. 
We define the mapping $\psi: \mathbf{M} \rightarrow T \mathbf{M}$ by

$$
\begin{aligned}
\psi(A) & =\operatorname{grad}_{A} \int_{0}^{\infty} W\left(s, A^{-1}: C^{0}(-s), A: C^{0^{-1}}(-s)\right) d s \\
& =\int_{0}^{\infty} h\left(s, A, C^{0}(-s)\right) d s \in T_{A} \mathbf{M}
\end{aligned}
$$

where $h(s, A, B)=\operatorname{grad}_{A} W\left(s, A^{-1}: B, A: B^{-1}\right)$ is as defined in $\S 4$. Then $\psi$ is the gradient of the functional

$$
\Psi(A)=\int_{0}^{\infty} H\left(s, A, C^{0}(-s)\right) d s .
$$

Let $C^{\eta}$ be a solution of $\left(16_{\eta}\right)$ for some $\eta>0$. Then in $T_{C^{\eta}(t)} \mathbf{M}$ for $t \geq 0$

$$
\begin{aligned}
0= & \eta C^{\eta^{\prime}}(t)+\int_{-\infty}^{t} h\left(t-s, C^{\eta}(t), C^{\eta}(s)\right) d s \\
= & \eta C^{\eta^{\prime}}(t)+\psi\left(C^{\eta}(t)\right)+\int_{0}^{t} h\left(t-s, C^{\eta}(t), C^{\eta}(s)\right) d s \\
& +\int_{0}^{\infty}\left(h\left(t+s, C^{\eta}(t), C_{0}(-s)\right)-h\left(s, C^{\eta}(t), C_{0}(-s)\right)\right) d s .
\end{aligned}
$$

Now set $\widetilde{C}(\tau)=C^{\eta}(\eta \tau)$ (we drop the superscript, since it will always be clear from the context which value of $\eta$ is used), then in the new time variable $\tau$

$$
\widetilde{C}^{\prime}(\tau)+\psi(\widetilde{C}(\tau))=f^{\eta}(\eta \tau)
$$

with

$$
\begin{aligned}
f^{\eta}(\sigma)=\int_{0}^{\sigma} & h\left(\sigma-s, C^{\eta}(\sigma), C^{\eta}(s)\right) d s \\
& +\int_{0}^{\infty}\left(h\left(\sigma+s, C^{\eta}(\sigma), C_{0}(-s)\right)\right. \\
& \left.-h\left(s, C^{\eta}(\sigma), C_{0}(-s)\right)\right) d s
\end{aligned}
$$

We assume now that $(\mathrm{H} 0),(\mathrm{H} 2)$ and $(\mathrm{H} 3)$ hold, and first investigate the solution behavior of the ordinary differential equation

$$
D^{\prime}(\tau)+\psi(D(\tau))=0 \quad \text { in } T_{D(\tau)} \mathbf{M} \quad(\tau \geq 0)
$$

that results from setting $f^{\eta}(\cdot)=0$ in (49).

LEMMA 7.1. For any $D(0) \in \mathbf{M}$, equation (51) has a unique solution on $[0, \infty)$, and there exist constants $K, \varepsilon>0$ such that

$$
\left\|D(\tau)-C^{00}\right\| \leq K e^{-\varepsilon \tau} \quad(\tau \geq 0),
$$


where $C^{00}$ is the unique minimum of $\Psi$ on $\mathbf{M}$, i.e. the initial value of $C^{0}(\cdot)$.

Proof. Local existence and uniqueness follow from the smoothness assumptions made in ( $\mathrm{H} 0)$. Moreover,

$$
\frac{d}{d \tau} \Psi(D(\tau))=-\|\psi(D(\tau))\|_{D(\tau)}^{2},
$$

implying that the Lyapunov function $\Psi(D(\tau))$ must decrease as long as the solution is not stationary. Thus the solution must exist for all times, and its $\omega$-limit set must be contained in the set of stationary points of $\Psi$. By Lemma 6.2, $\Psi$ has the unique minimum $C^{00}$ and no other stationary points. Thus the solution $D(\cdot)$ must tend to the limit $C^{00}$. The estimate (52) can be obtained by forming the covariant derivative of (51) (which exists due to (H0)) and multiplying with $D^{\prime}(\tau)$. Using (39), this gives

$$
\frac{1}{2} \frac{d}{d \tau}\left\|D^{\prime}(\tau)\right\|_{D(\tau)}^{2}+\varepsilon\left\|D^{\prime}(\tau)\right\|_{D(\tau)}^{2} \leq 0,
$$

and (52) follows.

THEOREM 7.2. Let $\psi$ be defined as above, and assume that (H0)(H3) hold. Let $C^{\eta}(\cdot)$ be the solution of $\left(17_{\eta}\right)$ and $D(\cdot)$ be the solution of (51). Then there exist constants $K, L>0$, depending on the data, but not on $\eta$, such that for $0 \leq t \leq 1$ and all small positive $\eta$ the following estimates hold:

$$
\begin{gathered}
\left\|C^{\eta}(t)-D\left(\frac{t}{\eta}\right)\right\| \leq K \frac{t^{2}}{\eta} e^{L t / \eta}, \\
\left\|\frac{d}{d t} C^{\eta}(t)\right\| \leq \frac{K}{\eta}\left(\frac{t^{2}}{\eta} e^{L t / \eta}+t+e^{-\varepsilon t / \eta}\right),
\end{gathered}
$$

where $\varepsilon$ is as in (52).

Proof. The equations (49) and (51) can also be interpreted as ordinary differential equations in the linear space of symmetric $3 \times 3$ matrices, if the pressure term contained in $\psi$ is eliminated as in $\S 4$. We now recall that by Theorem 5.1(c) the $C^{\eta}$ are uniformly bounded, independent of $\eta$. Therefore

$$
\|\psi(\widetilde{C}(\tau))-\psi(D(\tau))\| \leq L\|\widetilde{C}(\tau)-D(\tau)\|
$$


for all $\tau$ and $\eta$, with a uniform Lipschitz constant $L>0$. Also, inspection of (50) together with ( $\mathrm{H} 0)$ and the uniform bound for the $C^{\eta}$ shows that for some $K$ that does not depend on $\eta$

$$
\left\|f^{\eta}(\eta \tau)\right\| \leq \eta K \tau
$$

for $0 \leq \tau \leq 1 / \eta$. Thus by (49) and (51)

$$
\begin{aligned}
\|\widetilde{C}(\tau)-D(\tau)\| & \leq \int_{0}^{\tau}\left\|\tilde{C}^{\prime}(\sigma)-D^{\prime}(\sigma)\right\| d \sigma \\
& \leq \int_{0}^{\tau}(L\|\widetilde{C}(\sigma)-D(\sigma)\|+\eta K \sigma) d \sigma .
\end{aligned}
$$

Solving this integral inequality gives

$$
\|\widetilde{C}(\tau)-D(\tau)\| \leq K \eta \tau^{2} e^{L \tau}
$$

for $0 \leq \tau \leq 1 / \eta$ and for some $K_{2}>0$. Replacing $\tau$ with $t / \eta$ in (58) results in (54).

To prove (55), we estimate

$$
\begin{aligned}
\eta\left\|\frac{d}{d t} C^{\eta}(t)\right\| \leq & \eta\left\|\frac{d}{d t} C^{\eta}(t)-\frac{d}{d t} D\left(\frac{t}{\eta}\right)\right\|+\eta\left\|\frac{d}{d t} D\left(\frac{t}{\eta}\right)\right\| \\
\leq & \left\|\psi\left(C^{\eta}(t)\right)-\psi\left(D\left(\frac{t}{\eta}\right)\right)\right\| \\
& +\left\|f^{\eta}(t)\right\|+\left\|\psi\left(D\left(\frac{t}{\eta}\right)\right)-\psi(D(\infty))\right\| \\
& \leq L K \frac{t^{2}}{\eta} e^{L t / \eta}+K t+K e^{-\varepsilon t / \eta},
\end{aligned}
$$

which is (55).

Theorem 7.2 implies that for small $\eta$ and for times $t \leq O(|\eta \log \eta|)$ the material sample behaves approximately like a viscoelastic solid. We note that (54) and (55) imply in particular estimates of the form

$$
\begin{array}{cc}
\left\|C^{\eta}(t)-D\left(\frac{t}{\eta}\right)\right\| \leq K \eta^{\alpha} & \left(0 \leq t \leq t_{0}(\eta)\right), \\
\left\|C^{\eta}(t)-C^{0}(0)\right\| \leq K \eta^{\alpha} & \left(t_{0}(\eta) \leq t \leq 2 t_{0}(\eta)\right)
\end{array}
$$

where $t_{0}(\eta)=c \eta|\log \eta|$, the small constant $c$ is fixed, $\alpha>0$, and $K>0$ is independent of $\alpha$ and $\eta$. Also, by choosing $c$ sufficiently small, we can guarantee that

$$
\left\|\frac{d}{d t} C^{\eta}(t)\right\| \leq K \eta^{-\beta} \quad\left(t_{0}(\eta) \leq t \leq 2 t_{0}(\eta)\right),
$$


and $\beta>0$ can be made as close to zero as we please. These estimates are considerably weaker than those given in [11] for the scalar equation, where e.g. the right hand side of (54) is replaced by $K t+O(\eta)$, leading to right hand sides $K \eta|\log \eta|$ in $(59 . \mathrm{a}, \mathrm{b})$. With some additional effort, such estimates can probably also be proved for the problem under consideration; however, in the next section, only the weaker results given here will be used.

8. Interior estimates. The purpose of this section is to show that $C^{\eta}(t) \rightarrow C^{0}(t)$ as $t \rightarrow \infty$, uniformly on any set that is bounded away from 0 , at an algebraic rate in $\eta$ (see (61)). Together with (59), this gives a complete description of the behavior of $C^{\eta}(t)$ for $0 \leq t<\infty$, as $\eta \downarrow 0$, up to terms of order $\eta^{\alpha}$. We introduce the additional hypothesis

(H4) For any $R>0$ there exist $K=K_{R}>0$ and $m(\cdot)=m_{R}(\cdot) \in$ $L^{1}(0, \infty ; \mathbf{R})$ such that

$$
\begin{aligned}
-m_{R}(s) & \leq W^{\prime \prime}\left(s, \lambda_{1}, \lambda_{2}\right) \\
& \leq-K W^{\prime}\left(s, \lambda_{1}, \lambda_{2}\right) \quad\left(0 \leq s<\infty, 3 \leq \lambda_{i} \leq R\right) .
\end{aligned}
$$

We note that $(\mathrm{H} 2)$ and $(\mathrm{H} 4)$ together imply that

$$
-K W\left(s, \lambda_{1}, \lambda_{2}\right) \leq W^{\prime}\left(s, \lambda_{1}, \lambda_{2}\right) \leq-\delta W\left(s, \lambda_{1}, \lambda_{2}\right)
$$

with $K$ depending on the size of $\lambda_{1}, \lambda_{2}$.

TheOREM 8.1. Let (H0), (H2), (H3) and (H4) hold. Then for any $c>0$ there exist $\alpha>0, K>0$ such that

$$
\sup _{t \geq c \eta|\log \eta|}\left\|\dot{C}^{\eta}(t)-C^{0}(t)\right\| \leq K \eta^{\alpha} .
$$

For the proof, we need some estimates for the norm of $C^{\eta^{\prime}}(t)$ on the intervals on which (61) is claimed to hold. These are given in the following lemma. In its statement and proof all norms $\left\|C^{\eta^{\prime}}(t)\right\|$ are understood to be evaluated at $C^{\eta}(t)$.

LEMMA 8.2. With the assumptions and notation of Theorem 8.1 and $\delta>0$ as in (H2), there exist constants $\varepsilon, K>0$, independent of $\eta$, such that for all $0 \leq t_{0} \leq t_{1}<\infty$

$$
\eta e^{\delta t_{1}}\left\|C^{\eta^{\prime}}\left(t_{1}\right)\right\|^{2}+\varepsilon \int_{t_{0}}^{t_{1}} e^{\delta s}\left\|C^{\eta^{\prime}}(s)\right\|^{2} d s \leq K+\eta e^{\delta t_{0}}\left\|C^{\eta^{\prime}}\left(t_{0}\right)\right\|^{2}
$$

Proof. The right hand side of $\left(16_{\eta}\right)$ is absolutely continuous, hence $C^{\eta^{\prime \prime}}$ exists almost everywhere. We differentiate $\left(16_{\eta}\right)$ covariantly, 
form the inner product in $T_{C^{\eta}(t)} \mathbf{M}$ with $C^{\eta^{\prime}}(t)$, and multiply the result with $e^{\delta t}$, where $\delta$ is as in (H2). This gives the identity

$$
\begin{aligned}
\frac{d}{d t}\left(\frac{\eta}{2} e^{\delta t}\left\|C^{\eta^{\prime}}(t)\right\|^{2}+e^{\delta t} \int_{-\infty}^{t} H^{\prime}\left(t-s, C^{\eta}(t), C^{\eta}(s)\right) d s\right) \\
\quad+e^{\delta t} \int_{-\infty}^{t}\left\langle\nabla_{C^{\eta^{\prime}}(t)} \operatorname{grad}_{A} h\left(t-s, C^{\eta}(t), C^{\eta}(s)\right), C^{\eta^{\prime}}(t)\right\rangle_{C^{\eta}(t)} d s \\
=e^{\delta t}\left(\frac{\eta \delta}{2}\left\|C^{\eta^{\prime}}(t)\right\|^{2}+\int_{-\infty}^{t}\left(H^{\prime \prime}\left(t-s, C^{\eta}(t), C^{\eta}(s)\right)\right.\right. \\
\left.\left.+\delta H^{\prime}\left(t-s, C^{\eta}(t), C^{\eta}(s)\right)\right) d s\right) .
\end{aligned}
$$

By Lemma 6.2, the last term on the left hand side of this identity can be estimated from below by $\varepsilon\left\|C^{\eta^{\prime}}(s)\right\|$, where $\varepsilon>0$ is some constant, independent of $\eta$ due to the uniform bound for the $C^{\eta}(\cdot)$ that was established in Theorem 5.1. For the same reason we can apply (H4) to estimate the right hand side of this identity from above by

$$
e^{\delta t}\left(\frac{\eta \delta}{2}\left\|C^{\eta^{\prime}}(t)\right\|^{2}-K \int_{-\infty}^{t} H^{\prime}\left(t-s, C^{\eta}(t), C^{\eta}(s)\right) d s\right) .
$$

Now integrate the resulting inequality between $t_{0}$ and $t_{1}$ to obtain

$$
\begin{aligned}
& \eta e^{\delta t_{1}}\left\|C^{\eta^{\prime}}\left(t_{1}\right)\right\|^{2}-e^{\delta t_{0}}\left\|C^{\eta^{\prime}}\left(t_{0}\right)\right\|^{2}+\varepsilon \int_{t_{0}}^{t_{1}} e^{\delta s}\left\|C^{\eta^{\prime}}(s)\right\|^{2} d s \\
& \leq \int_{t_{0}}^{t_{1}} e^{\delta s}\left(\eta \delta\left\|C^{\eta^{\prime}}(t)\right\|^{2}\right. \\
& \left.\quad-2 K \int_{-\infty}^{t} H^{\prime}\left(t-s, C^{\eta}(t), C^{\eta}(s)\right) d s\right) d t .
\end{aligned}
$$

By estimate (30), the right hand side is bounded uniformly in $t_{0}, t_{1}$, and $\eta$, and the lemma follows.

Proof of Theorem 8.1. For any $t>0, C^{0}(t)$ is the unique minimizer of the functional

$$
A \rightarrow G^{0}(t, A)=\int_{-\infty}^{t} H\left(t-s, A, C^{0}(s)\right) d s,
$$

while $C^{\eta}(t)$ is a critical point of the functional

$$
\begin{aligned}
A \rightarrow G_{1}^{\eta}(t, A) & =G^{\eta}(t, A)+\eta C^{\eta^{\prime}}(t): A, \\
G^{\eta}(t, A) & =\int_{-\infty}^{t} H\left(t-s, A, C^{\eta}(s)\right) d s,
\end{aligned}
$$


cf. (32) and Lemma 6.2. Let $C_{1}^{\eta}(t)$ be the unique minimizer of the functional $G^{\eta}(t, \cdot)$; since the $C^{\eta}(\cdot)$ are uniformly bounded, so are the $C_{1}^{\eta}(\cdot)$. Then by Lemma $6.2(\mathrm{c})$

$$
\left\|C^{0}(t)-C_{1}^{\eta}(t)\right\| \leq \int_{0}^{t} m_{R}(t-s)\left\|C^{0}(s)-C^{\eta}(s)\right\| d s
$$

for all $0 \leq t<\infty$, with $m_{R}(\cdot) \in\left(L^{1} \cap L^{\infty}\right)(0, \infty)$. Next, by Lemma 8.2 and $(60)$,

$$
\eta\left\|C^{\eta^{\prime}}(t)\right\| \leq \eta^{1 / 2} e^{-\delta t / 2}\left(K_{0}+K_{1} \eta^{-\beta}\right),
$$

whenever $t \geq t_{0}(\eta)=c \eta|\log \eta|$, where $\beta>0$ can be made as small as we please by choosing $c>0$ small. Picking $c$ so small that $\beta<1 / 2$, this estimate implies

$$
\eta\left\|C^{\eta^{\prime}}(t)\right\| \leq K \eta^{\kappa} e^{-\delta t / 2}
$$

for $t \geq t_{0}(\eta)$, with $K, \kappa, \delta>0$ independent of $\eta$. Consider now the full functional $G_{1}^{\eta}(t, \cdot)$. Lemma 6.2 implies that for all sufficiently small $\eta$ and for all $t \geq t_{0}(\eta)$, an estimate of the form (39) holds for this functional in a sufficiently large ball in $\mathbf{M}$ in which all solutions live. Thus $C^{\eta}(t)$ is the unique minimizer of $G_{1}^{\eta}(t, \cdot)$ in this ball, and we can estimate with some $\rho>0$

$$
\begin{aligned}
G_{1}^{\eta}(t, & \left.C^{\eta}(t)\right)=G^{\eta}\left(t, C^{\eta}(t)\right)+\eta C^{\eta^{\prime}}(t): C^{\eta}(t)=G^{\eta}\left(t, C^{\eta}(t)\right) \\
\leq & G^{\eta}\left(t, C_{1}^{\eta}(t)\right)-\rho\left\|C_{1}^{\eta}(t)-C^{\eta}(t)\right\|^{2} \\
\leq & G^{\eta}\left(t, C_{1}^{\eta}(t)\right)+\eta C^{\eta^{\prime}}(t): C_{1}^{\eta}(t) \\
& -\rho\left\|C_{1}^{\eta}(t)-C^{\eta}(t)\right\|^{2}+K \eta^{\kappa} \\
\leq & G^{\eta}\left(t, C^{\eta}(t)\right)+\eta C^{\eta^{\prime}}(t): C^{\eta}(t) \\
& -2 \rho\left\|C_{1}^{\eta}(t)-C^{\eta}(t)\right\|^{2}+K \eta^{\kappa} \\
& =G_{1}^{\eta}\left(t, C^{\eta}(t)\right)-2 \rho\left\|C_{1}^{\eta}(t)-C^{\eta}(t)\right\|^{2}+K \eta^{\kappa}
\end{aligned}
$$

and thus

$$
\left\|C^{\eta}(t)-C_{1}^{\eta}(t)\right\| \leq K \eta^{\kappa / 2}
$$

for all $t \geq t_{0}(\eta)$. Combining this estimate with (67) gives

$$
\left\|C^{0}(t)-C^{\eta}(t)\right\| \leq K \eta^{\kappa / 2}+\int_{0}^{t} m_{R}(t-s)\left\|C^{0}(s)-C^{\eta}(s)\right\| d s
$$

for $t \geq t_{0}(\eta)$. This integral inequality implies

$$
\left\|C^{0}(t)-C^{\eta}(t)\right\| \leq K \eta^{\kappa / 2} e^{L t}
$$


for some fixed constants $K, L$, for all $t \geq t_{0}(\eta)$. On the other hand, by the $L^{2}$-estimate for $\left\|C^{\eta^{\prime}}(\cdot)\right\|$ in Lemma 8.2 and by the proof of Lemma 6.4, we have

$$
\left\|C^{\eta}(t)-C^{\eta}(s)\right\| \leq K e^{-\sigma s}
$$

for all $t \geq s \geq t_{0}(\eta)$ and also for $\eta=0, t \geq s \geq 0$, for some fixed $K, \sigma>0$. Combining (72) and (73) gives

$$
\left\|C^{\eta}(t)-C^{0}(t)\right\| \leq 2 K e^{-\sigma s}+K \eta^{\kappa / 2} e^{L s}
$$

from which the theorem follows by choosing

$$
s=\frac{\kappa}{2(L+\sigma)}|\log \eta| \text { and } \alpha=\frac{\sigma \kappa}{2(\sigma+L)} .
$$

9. Qualitative behavior. In this section, some qualitative properties for the finite time and asymptotic behavior of solutions of $\left(16_{\eta}\right)$ and $\left(16_{0}\right)$ will be derived. In particular, these results will be used in the next section to show that under suitable assumptions a shear-free deformation history always leads to a shear-free recovery and that a simple shear or pure shear history can never lead to a recovery in the same deformation class. Some qualitative results from [11] are also recovered.

THEOREM 9.1. Let $\mathbf{V} \subset \mathbf{R}^{3,3}$ be a linear space such that $A B^{-1} A \in$ $\mathbf{V}$ whenever $A, B \in \mathbf{V} \cap \mathbf{M}$. Let the assumptions (H0)-(H4) hold, let $\eta \geq 0$, and assume that $C^{\eta}(\cdot)$ is a solution of $\left(16_{\eta}\right)$ with data $C_{0}(\cdot), C_{00} \in \mathbf{V}$. Then $C^{\eta}(t) \in \mathbf{V} \quad(0 \leq t<\infty)$.

Proof. Let $q(\cdot)$ denote the seminorm given by $q(A)=\operatorname{dist}(A, \mathbf{V})$ for symmetric $3 \times 3$-matrices $A$. Then $q(A B) \leq K(A) q(B)$ for some constant $K(A)$. Now let $u(t)=q\left(C^{\eta}(t)\right)$, and assume first that $\eta>0$. Integrating the integrodifferential equation $\left(16_{\eta}\right)$ once with respect to $t$ and applying $q(\cdot)$ to the result gives an integral inequality of the form

$$
\eta u(t) \leq \int_{0}^{t} a(t, s) u(s) d s
$$

with $a(\cdot, \cdot)$ depending on the solution itself, since $q\left(C_{0}(\cdot)\right)=0$, $q\left(C_{00}\right)=0$ by assumption. By Gronwall's lemma, $u(t)=0$, i.e. $C^{\eta}(t) \in \mathbf{V}$ for all $t>0$. If $\eta=0$, then $C^{0}(\cdot)$ can be approximated uniformly by solutions $C^{\eta}(\cdot)$ that remain in $\mathbf{V}$, by the results of $\S \S 7$ and 8. Thus also $C^{0}(\cdot)$ remains in $\mathbf{V}$. 
COROllaRY 9.2. If $W$ does not depend on its last argument, then the solution $C^{\eta}(\cdot)$ will be in the linear space spanned by a.e. $C_{0}(\cdot)$ (if $\eta=0$ ) resp. by a.e. $C_{0}(\cdot)$ and $C_{00}$ (if $\eta>0$ ). If $W$ does not depend on its second argument, then the solutions $C^{\eta}(\cdot)$ will satisfy $\left(C^{\eta}(\cdot)\right)^{-1} \in V_{1}$, where $V_{1}$ is the linear space spanned by a.e. $C_{0}^{-1}(\cdot)$ (if $\eta=0$ ) resp. by a.e. $C_{0}^{-1}(\cdot)$ and by $C_{00}^{-1}($ if $\eta>0)$.

Proof. The first assertion can be proved in the same way as Theorem 9.1. The second statement follows from the first since $D^{\eta}(\cdot)=$ $\left(C^{\eta}(\cdot)\right)^{-1}$ satisfies $\left(16_{\eta}\right)$ with $W\left(s, \lambda_{1}, \lambda_{2}\right)$ replaced by $W\left(s, \lambda_{2}, \lambda_{1}\right)$ and with data $C_{0}^{-1}(\cdot), C_{00}^{-1}$.

The second main result of this section will allow us to prove that for certain deformation histories, the material sample will never recover to its original shape or to any shape it has previously been in. These surprising effects, which are discussed in more detail for a special constitutive relation in [9], will be explained in the next section. An abstract version of Theorem 9.3 below states that if $K \subset \mathbf{R}^{3,3}$ is the closed convex hull of $\left\{C_{0}(t) \mid t<0\right\} \cup\left\{C_{00}\right\} \cup\{0\}$, then for $0<t \leq \infty$ the solution $C^{\eta}(t)$ will be in the interior of $\mathbf{K}$ (relative to the linear space spanned by $\mathbf{K}$ ). We prefer to state this property in the form of inequalities for components of the solution.

The following hypothesis will be needed.

(H5)(i) $W:[0, \infty) \times[3, \infty) \times[3, \infty) \rightarrow \mathbf{R}$ does not depend on its last argument.

(ii) $W_{11}(s, \lambda) \leq K_{R} W_{1}(s, \lambda)$, if $3 \leq \lambda \leq R$.

(iii) $W_{1}(s, 3)>0$ for a.e. $s>0$.

THEOREM 9.3. Let the assumptions (H0)-(H3) and (H5) hold. Let $C^{\eta}(\cdot)$ be the solution of $\left(16_{\eta}\right)$ for $\eta \geq 0$. Let $D \in \mathbf{R}^{3,3}$. If $\eta>0$, we assume that $\left\{t \mid C_{0}(t) \neq C_{00}\right\}$ is not a null set and that $\left|D: C_{0}(\cdot)\right|+$ $\left|D: C_{00}\right|$ is not identically zero. If $\eta=0$, we assume that $C_{0}(\cdot)$ is not a.e. identically equal to a constant matrix and that $\left|D: C_{0}(\cdot)\right|$ is not identically zero. Let $\alpha_{0} \leq 0 \leq \alpha_{1}$ be numbers such that

$$
\begin{array}{ll}
\alpha_{0} \leq C_{0}(s): D \leq \alpha_{1} & (s<0), \\
\alpha_{0} \leq C_{00}: D \leq \alpha_{1}, & \text { if } \eta>0 .
\end{array}
$$

Then

$$
\alpha_{0}<C^{\eta}(t): D<\alpha_{1} \quad(t>0)
$$


and

$$
\alpha_{0}<\lim _{t \rightarrow \infty} C^{\eta}(t): D<\alpha_{1}
$$

Note that the existence of the limit in (76) follows from previous results. For the proof, we need some simple auxiliary estimates. If (H5) (i) holds, then $\left(16_{\eta}\right)$ has the quasilinear form

$$
\eta C^{\eta^{\prime}}(t)+p(t) C^{\eta}(t)=\int_{-\infty}^{t} b(t, s) C^{\eta}(s) d s
$$

with

$$
\begin{aligned}
b(t, s) & =W_{1}\left(t-s, \lambda^{\eta}(t, s)\right), \\
p(t) & =\int_{-\infty}^{t} b_{0}(t, s) d s, \\
b_{0}(t, s) & =W_{1}\left(t-s, \lambda^{\eta}(t, s)\right) \frac{\lambda^{\eta}(t, s)}{3} \text { and } \\
\lambda^{\eta}(t, s) & =C^{\eta-1}(t): C^{\eta}(s) .
\end{aligned}
$$

We also abbreviate $a(s)=W_{1}(s, 3)$ and $\bar{a}(t)=\int_{t}^{\infty} a(s) d s$.

LEMMA 9.4. With all assumptions and notations as above, the following estimates hold for $0 \leq s, t, \tau<\infty, 3 \leq \lambda \leq R$, with $\delta>0$ as in $(\mathrm{H} 2)$ and $\varepsilon=\varepsilon(R)>0, K_{0}=K(R)>0$.

$$
\begin{aligned}
b(s, \tau) & \geq a(\tau), \\
a(s) & \geq K_{0} e^{K_{0}(3-\lambda)} W(s, \lambda), \\
\bar{a}(t) & \geq \varepsilon e^{-\delta t}, \\
0 & \leq p(t)-\bar{a}(0) \leq K_{0} e^{-\delta t} .
\end{aligned}
$$

Proof. The first estimate follows directly from (H3). By (H5) (ii),

$$
e^{K_{0}(\lambda-3)} a(s) \geq W_{1}(s, \lambda)
$$

and therefore, since $W(s, 3)=0$,

$$
K_{0}^{-1} e^{K_{0}(\lambda-3)} a(s) \geq W(s, \lambda),
$$

which is (79). Integrating with respect to $s$ and using (H2) implies 
(80). Finally, by $(\mathrm{H} 5)(\mathrm{ii})$ and $(\mathrm{H} 2)$

$$
\begin{aligned}
0 & \leq p(t)-\bar{a}(0) \\
& =\int_{-\infty}^{t}\left(W_{1}\left(t-s, \lambda^{\eta}(t, s)\right) \frac{\lambda^{\eta}(t, s)}{3}-W_{1}(t-s, 3)\right) d s \\
& =\frac{1}{3} \int_{-\infty}^{t} \int_{3}^{\lambda^{\eta}(t, s)} \frac{d}{d \xi}\left(W_{1}(t-s, \xi) \xi\right) d \xi d s \\
& \leq K_{0} \int_{-\infty}^{t} \int_{3}^{\lambda^{\eta}(t, s)} W_{1}(t-s, \xi) d \xi d s \\
& =K_{0} \int_{-\infty}^{t} W\left(t-s, \lambda^{\eta}(t, s)\right) d s \leq K_{0} e^{-\delta t},
\end{aligned}
$$

which proves $(81)$.

Proof of Theorem 9.3. We suppress the dependence on $\eta$ and set $u(t)=C^{\eta}(t): D$. Then

$$
\eta u^{\prime}(t)=\int_{-\infty}^{t}\left(b(t, s) u(s)-b_{0}(t, s) u(t)\right) d s .
$$

We first prove (75) for the case $\eta>0$ by means of a standard comparison argument. Assume, e.g., that $u(t)=\alpha_{1}$ for some $t \geq 0$ and that $u(s) \leq \alpha_{1}$ for all $s \leq t, u(\cdot)$ not identically zero on $(-\infty, t]$. If $\alpha_{1}=0$, then the right hand side equals $\int_{-\infty}^{t} b(t, s) u(s) d s \leq$ $\int_{-\infty}^{t} a(t-s) u(s) d s<0$. Similarly, if $\alpha_{1}>0$, then the right hand side can be estimated by $\int_{-\infty}^{t}\left(b(t, s)-b_{0}(t, s)\right) d s \cdot \alpha_{1}$. This quantity must be negative, since $C^{\eta}(s)$ is not identically equal to $C^{\eta}(t)$ for $s \leq t$, therefore $\lambda^{\eta}(t, s)$ is not identically equal to 3 , therefore $b(t, s)<b_{0}(t, s)$ for $s$ in a set with positive measure. Thus in either case $u^{\prime}(t)<0$ together with $u(t)=\alpha_{1}$, and this is only possible if $t=0$. Therefore $u(t)<\alpha_{1}$ for all $t>0$, and similarly $u(t)>\alpha_{0}$ for all $t>0$.

Consider now the case $\eta=0$. Then $u$ satisfies the Volterra integral equation

$$
u(t)=\int_{-\infty}^{t} \frac{b(t, s)}{p(t)} u(s) d s
$$

for all $t \geq 0$. Assume again that $u(t)=\alpha_{1}$ for some $t, u(s) \leq \alpha_{1}$ for $s \leq t$. If $\alpha_{1}=0$, then the right hand side of this equation must be negative by assumption, a contradiction. If $\alpha_{1}>0$, then, since $C^{\eta}(\cdot)$ 
is not identically constant on $(-\infty, t]$, we have $\int_{-\infty}^{t} b(t, s) / p(t) d s<$ 1 , and

$$
\alpha_{1}=u(t)=\int_{-\infty}^{t} \frac{b(t, s)}{p(t)} u(s) d s \leq \int_{-\infty}^{t} \frac{b(t, s)}{p(t)} d s \cdot \alpha_{1}<\alpha_{1},
$$

again a contradiction. Thus $u(t)<\alpha_{1}$ for all $t \geq 0$, and by the same argument $u(t)>\alpha_{0}$ for all $t \geq 0$. Estimate (75) has been proved.

We now turn to the estimate (76), which will be proved by means of the same argument both for $\eta>0$ and for $\eta=0$. Assume, e.g., that $\lim _{t \rightarrow \infty} u(t)=\alpha_{1}$. Set $w(t)=u(t)-\alpha_{1}$, then by assumption $w(t) \leq 0$ for all $t$ and $w(t)<0$ on some subset of $(-\infty, 0)$ with positive measure. Set $c=\varepsilon \int_{0}^{\infty} e^{-\delta s} w(-s) d s<0$, where $\varepsilon$ and $\delta$ are as in (80). Since $\alpha_{1} \geq 0$,

$$
\eta w^{\prime}(t)+p(t) w(t) \leq \int_{-\infty}^{t} b(t, s) w(s) d s
$$

We want to derive a contradiction to the assumption that $\lim _{t \rightarrow \infty} w(t)$ $=0$. For this purpose, let $v:[T, \infty) \rightarrow \mathbf{R}$ be the unique solution of the problem

(85) $\eta v^{\prime}(t)+p(t) v(t)$

$$
=\int_{-\infty}^{t} b(t, s) v(s) d s \quad(t \geq T), \quad v(t)=w(t) \quad(t \leq T)
$$

where $T>0$ will be chosen below. A comparison argument, using that $b(t, s) \geq a(t-s)>0$, shows that $w(t) \leq v(t) \leq 0$ for all $t \geq T$ and hence also $v(t) \rightarrow 0$ as $t \rightarrow \infty$. We integrate (85) from $T$ to $T+s>T$ and obtain after rearranging terms and using the definition of $v$ for $t \leq T$

$$
\begin{aligned}
\eta v(T+ & +s)+\int_{0}^{s} \bar{a}(s-\tau) v(T+\tau) d \tau \\
& +\int_{0}^{s}(p(T+\tau)-\bar{a}(0)) v(T+\tau) d \tau \\
& =\int_{-\infty}^{T}(\bar{a}(T-\tau)-\bar{a}(T+s-\tau)) w(\tau) d \tau+\eta w(T) .
\end{aligned}
$$

We now choose $T$ so large that for all $t \geq T$

$$
\frac{c \delta}{2 K_{0}} \leq w(t) \leq v(t) \leq 0
$$

where $\delta$ and $c$ are as above and $K_{0}$ is as in (81). Then

$$
\begin{aligned}
& \int_{0}^{s}(p(T+\tau)-\bar{a}(0)) v(T+\tau) d \tau \\
& \quad \geq-\int_{0}^{\infty} K_{0} e^{-\delta(T+\tau)} \frac{c \delta}{2 K_{0}} d \tau \geq \frac{c}{2} e^{-\delta T},
\end{aligned}
$$


and thus the limes inferior (as $s \rightarrow \infty$ ) of the left hand side of (86) is bounded below by $\frac{c}{2} e^{-\delta T}$. On the other hand, the right hand side of (86) can be estimated from above by

$$
\begin{gathered}
\int_{-\infty}^{T} \varepsilon e^{-\delta(T-\tau)} w(\tau) d \tau-\int_{-\infty}^{T} \bar{a}(T+s-\tau) w(\tau) d \tau \\
\leq c e^{-\delta T}-\int_{0}^{\infty} \bar{a}(s+\tau) w(T-\tau) d \tau .
\end{gathered}
$$

Taking the limes superior of this expression as $s \rightarrow \infty$, we arrive at the contradiction

$$
\frac{c}{2} e^{-\delta T} \leq c e^{-\delta T}
$$

Therefore, $w(\cdot)$ cannot go to 0 as $t \rightarrow \infty$, and $\lim _{t \rightarrow \infty} u(t)$ must be strictly less than $\alpha_{1}$. By the same argument, $\lim _{t \rightarrow \infty} u(t)>\alpha_{0}$. The theorem is completely proved.

\section{Applications to rheological models.}

Examples of $K$-BKZ-models. We first conisder the rubberlike liquid model ([9]), for which

$$
W\left(s, \lambda_{1}, \lambda_{2}\right)=m_{1}(s) \lambda_{1}
$$

and the Ward-Jenkins-model ([20]), with

$$
W\left(s, \lambda_{1}, \lambda_{2}\right)=m_{1}(s) \lambda_{1}+m_{2}(s) \lambda_{2} \text {. }
$$

Here the kernels $m_{i}(\cdot)$ have the form

$$
m_{i}(s)=\sum_{j=1}^{N} a_{i j} e^{-s / \tau_{i j}}, \quad a_{i j} \geq 0, \tau_{i 1}>\tau_{i 2}>\cdots>0 .
$$

Obviously, hypotheses (H0)-(H4) hold for both models, and (H5) holds for the rubberlike liquid model. Similarly, these hypotheses hold for a number of phenomenological models of K-BKZ-type in which $W\left(s, \lambda_{1}, \lambda_{2}\right)=\sum_{j=1}^{n} m_{j}(s) U_{j}\left(\lambda_{1}, \lambda_{2}\right)$ with functions $U_{j}$ that are of polynomial or logarithmic type, such as Zapas' model ([21]).

Consider next the Doi-Edwards-model ([4]), for which in the notation of $\S 4$

$$
H(s, A, B)=\mu(s) U\left(B^{-1 / 2} A B^{-1 / 2}\right)=\mu(s) U\left(A^{1 / 2} B^{-1} A^{1 / 2}\right),
$$

with

$$
\mu(s)=K \sum_{k \text { odd }} e^{-s k^{2} / T}, \quad U(D)=\int_{|v|=1} \log \left(v^{T} D v\right)
$$


where $K, T>0$ are some material constants, according to calculations given in [3]. Since the kernel $\mu(\cdot)$ has a singularity at 0 , the theory developed here can only be applied if the sum is extended over finitely many terms. From the definition of $U(D)=U\left(B^{-1 / 2} A B^{-1 / 2}\right)$, it is clear that this function depends only on the spectrum of the matrix $D$, and thus this is indeed a K-BKZ-model. Assumptions ( $\mathrm{H} 0)-(\mathrm{H} 2)$ then are easy to check. Checking (H3), however, is quite awkward. It is easier to show directly that the crucial property (39) holds for $U$, as follows: Let $A, B \in \mathbf{M}$, let $A(\cdot)$ be a geodesic in $\mathbf{M}$ with $A(0)=A$ and $A^{\prime}(0)=C \in T_{A} \mathbf{M}$, i.e.

$$
A(t)=A^{1 / 2} \exp \left(t A^{-1 / 2} C A^{-1 / 2}\right) A^{1 / 2} .
$$

As before, let $B^{-1 / 2} A(0) B^{-1 / 2}=D$. Then

$$
\begin{aligned}
& \left\langle\nabla_{C} \operatorname{grad}_{A} U\left(B^{-1 / 2} A B^{-1 / 2}\right), C\right\rangle_{A}=\left.\frac{d^{2}}{d t^{2}} U\left(B^{-1 / 2} A(t) B^{-1 / 2}\right)\right|_{t=0} \\
& =\int_{|v|=1} \frac{\left(v^{T} B^{-1 / 2} C A^{-1} C B^{-1 / 2} v\right)\left(v^{T} D v\right)-\left(v^{T} B^{-1 / 2} C B^{-1 / 2} v\right)^{2}}{\left(v^{T} D v\right)^{2}} .
\end{aligned}
$$

By Cauchy-Schwarz, the integrand is non-negative and zero if and only if the vectors $A^{1 / 2} B^{-1 / 2} v$ and $A^{-1 / 2} C B^{-1 / 2} v$ are linearly dependent. This will only be true for all $v$ from the unit sphere of $\mathbf{R}^{3}$ if the matrix $A^{-1 / 2} C A^{-1 / 2}$ is a multiple of the unit matrix. But since $\operatorname{tr} A^{-1 / 2} C A^{-1 / 2}=0$, this means that $C=0$. Thus the integral above must be positive for all $C \neq 0$, and (39) holds. Since also (H4) holds if only finitely many terms are used in the definition of the kernel $\mu(\cdot)$, all results in $\S \S 5-8$ hold for this modified Doi-Edwards model.

Deformations with orthogonal imbedded planes or vectors. In the remainder of this section, we use the notation for special deformations that was introduced in $\S 1$. First, some classes of deformation histories will be identified which will always lead to a recoil in the same class, without assuming (H5). By Theorem 9.1, we have to identify linear spaces $\mathbf{V}$ of $3 \times 3$-matrices such that $A B^{-1} A \in \mathbf{V}$ whenever $A, B \in$ $\mathbf{V} \cap \mathbf{M}$. One such space consists of all symmetric matrices of the form

$$
A=\left(\begin{array}{lll}
* & * & 0 \\
* & * & 0 \\
0 & 0 & *
\end{array}\right)
$$

where $*$ stands for an arbitrary real number. Then Theorem 9.1 applies. By the interpretation of the deformation tensor $\gamma$ given in $\S 1$, this means that there exist a vector and a plane, embedded in the 
material, that remain orthogonal throughout the entire deformation history. Then also the recoil will have this property. Thus a deformation history consisting of shear-free deformations and simple shear will lead to deformations of the form above during recoil. All diagonal matrices in $\mathbf{R}^{3,3}$ also form an admissible space $\mathbf{V}$. Thus, if there are three embedded vectors that formed an orthogonal system for the entire deformation history, they will remain orthogonal during the recoil. Thus a shear-free history will always lead to a shear-free recoil. An even smaller space $\mathbf{V}$ is obtained, if an additional linear relation is to hold for the diagonal elements, as well as for their reciprocals. One can show that essentially the only such relation is that two diagonal elements be equal. This of course corresponds to sheet stretching and filament stretching, the case that was studied in [11].

If $W\left(s, \lambda_{1}, \lambda_{2}\right)$ does not depend on its last argument, then by Corollary 9.2 also the space $\mathbf{V}$ of all symmetric matrices of the form

$$
A=\left(\begin{array}{lll}
* & * & 0 \\
* & * & * \\
0 & * & *
\end{array}\right)
$$

can be used. This means that any two embedded material planes will remain orthogonal during the recoil, if they were so during the deformation history.

Deformations with constant plane separation. In the remainder of this section, hypothesis (H5) will be assumed, such that Theorem 9.3 can be applied. Consider now a deformation history, given by $(\gamma(t))_{t \leq 0}=\left(C^{-1}(t)\right)_{t \leq 0}$, for which a diagonal component of $C(\cdot)$ is constant (positive) on $(-\infty, 0]$, but for which $\gamma(\cdot)$ itself is not constant. This means that there are two parallel planes in the material sample whose separation remained constant throughout the entire deformation history. We claim that for all $t>0$ and also in the limit as $t \rightarrow \infty$ these planes will have a separation that is strictly larger. To prove this, assume that the two parallel planes are spanned by the vectors $e_{1}$ and $e_{2}$. Then their separation is given by $\left(\gamma^{33}(\cdot)\right)^{-1 / 2}=$ $\sqrt{C_{33}(\cdot)}$. By Theorem 9.3, if $C_{33}(\cdot)$ is constant and positive on $(-\infty, 0]$, it will be strictly less on $(0, \infty)$ and in the limit as $t \rightarrow \infty$; which proves the claim. Both pure shear and simple shear have this property. The result shows that after deformation histories in these classes, "the liquid will 'recover' to a state which it has never previously been in" ([9]). 
Elongational flows. We now specifically consider the cases of filament stretching and sheet stretching, where $y(\cdot)$ is the elongation ratio. Then $\gamma^{-1}(\cdot)=C(\cdot)$ has the same form as $\gamma(\cdot)$ (with $y^{-1}(t)$ instead of $y(t))$, and after a history of this form, the recoil will always lead to states of the same form, as was shown above. In the case of filament stretching, assume that for $-\infty<t \leq 0$

$$
1 \leq y(t) \leq \bar{y}=\sup _{t \leq 0} y(t)
$$

which corresponds to the case of filament stretching, and that $\bar{y}>1$. Then an application of Theorem 9.3 shows that $1<y(t)<\bar{y}$ for all $t>0$ and that $1<\lim _{t \rightarrow \infty} y(t)<\bar{y}$. Similarly, if for $-\infty<t \leq 0$

$$
\tilde{y}=\inf _{t \leq 0} y(t) \leq y(t) \leq 1
$$

and $\tilde{y}<1$ (the case of sheet stretching), then $\tilde{y}<y(t)<1$ for all $t>0$ and $\tilde{y}<\lim _{t \rightarrow \infty} y(t)<1$. These results were deduced in [11] under much more general assumptions.

Pure shear. We next consider deformation histories of the form (7) with $a(t)=x(t), b(t)=x^{-1}(t), c(t)=1$, and assume that for $-\infty<t \leq 0$

$$
1 \leq x(t) \leq \bar{x}=\sup _{t \leq 0} x(t)
$$

and that $\bar{x}>0$. By the same arguments as above, for $t>0$

$$
\gamma(t)=\left(\begin{array}{ccc}
a(t) & 0 & 0 \\
0 & b(t) & 0 \\
0 & 0 & c(t)
\end{array}\right)
$$

with the relation $a(t) b(t) c(t)=1$ and

$$
b(t)>\bar{x}^{-1}, \quad 1<c(t)<a(t)
$$

and consequently also $a(t)<\bar{x}$. These relations will also hold for the limit as $t \rightarrow \infty$.

Simple shear. We finally study the recoil of material samples following a deformation history of the form (8), with $a(\cdot)=s(\cdot), b(\cdot)=$ $c(\cdot)=0$, and

$$
0 \leq s(t) \leq \bar{s}=\sup _{t \leq 0} s(t) \quad(t \leq 0), \bar{s}>0 .
$$


Then for $t \leq 0$

$$
\gamma^{-1}=\left(\begin{array}{ccc}
1+s^{2} & -s & 0 \\
-s & 1 & 0 \\
0 & 0 & 1
\end{array}\right)
$$

By Theorem 9.1, therefore

$$
\gamma^{-1}=\left(\begin{array}{ccc}
a & -b & 0 \\
-b & c & 0 \\
0 & 0 & c
\end{array}\right), \quad \gamma=\left(\begin{array}{ccc}
c^{2} & b c & 0 \\
b c & c^{-1}+b^{2} & 0 \\
0 & 0 & c^{-1}
\end{array}\right),
$$

since also $c\left(a c-b^{2}\right)=1$. By Theorem 9.3, the inequalities

$$
\begin{aligned}
& 0<b<\bar{s}, \quad 0<c<1, \\
& a=c^{-2}+b^{2} c^{-1}<1+\bar{s}^{2}, \\
& -\bar{s}^{2}<a-\left(1+\bar{s}^{2}\right) c<0, \\
& b-\frac{\bar{s}}{1+\bar{s}^{2}} a<0
\end{aligned}
$$

hold for all $t>0$ and in the limit as $t \rightarrow \infty$. One reads off that the sample will expand in the $e_{2}$-direction and by a smaller amount also in the $e_{3}$-direction, and that it will contract in the $e_{1}$-direction. An upper bound for the expansion ratio in the $e_{2}$-direction can be obtained from the estimate

$$
c^{-1}+b^{2}=a c<1+\bar{s}^{2},
$$

implying that $c^{-1}+b^{2}<\sec \bar{\theta}$, where $\bar{\theta}=\sup _{t \leq 0} \theta(t)$ is the maximal shear angle and $\theta(\cdot)$ is defined as in $\S 1$. Also, the material slice between any two planes parallel to $\left(e_{1}, e_{3}\right)$ or to $\left(e_{1}, e_{2}\right)$ will expand by the same amount $c^{-1 / 2}<\sqrt{\sec \bar{\theta}}$. Similarly, since

$$
a=c^{-2}+b^{2} c^{-2}<1+\bar{s}^{2}+\frac{\bar{s}^{2}}{\sqrt{1+\bar{s}^{2}}}=\sec ^{2} \bar{\theta}+\sin \bar{\theta} \tan \bar{\theta},
$$

we have the universal lower bound $\left(\sec ^{2} \bar{\theta}+\sin \bar{\theta} \tan \bar{\theta}\right)^{-1 / 2}$ for the separation ratio of two planes that are parallel to $e_{2}$ and $e_{3}$ before and after recovery. In experiments, some lateral expansion of material samples has been observed; however, experimental data do not confirm that the two expansion ratios will be the same for the $\left(e_{1}, e_{3}\right)$ plane and for the $\left(e_{1}, e_{2}\right)$-plane, as predicted here (see [9]).

For the angle

$$
\theta=\cos ^{-1} \frac{b c}{\sqrt{c^{2}\left(c^{-1}+b^{2}\right.}}=\cos ^{-1} \frac{b}{\sqrt{a c}}
$$


between the vectors $e_{1}$ and $e_{2}$, the inequalities above imply the estimate

$$
\theta>\cos ^{-1} \frac{\sqrt{1+\bar{s}^{2}} b}{a}>\cos ^{-1} \frac{\bar{s}}{\sqrt{1+\bar{s}^{2}}}=\inf _{t<0} \theta(t) .
$$

\section{REFERENCES}

[1] J. S. Angell and W. E. Olmstead, Singular perturbation analysis of an integrodifferential equation modelling filament stretching, Zeitschr. Angew. Math. Physik, 36 (1985), 487-490.

[2] B. Bernstein, E. A. Kearsley and L. J. Zapas, A study of stress relaxation with finite strain, Trans. Soc. Rheology, 7 (1963), 391-410.

[3] P. K. Currie, Constitutive equations for polymer melts predicted by the DoiEdwards and Curtiss-Bird kinetic theory models, J. Non-Newtonian Fluid Mechanics, 11 (1982), 53-68.

[4] M. Doi and S. F. Edwards, The Theory of Polymer Dynamics, Clarendon Press, Oxford, 1986.

[5] M. E. Gurtin, An Introduction to Continuum Mechanics, Academic Press, 1981.

[6] S. Helgasson, Differential Geometry, Lie Groups, and Symmetric Spaces, Academic Press, London, New York, 1978.

[7] G. S. Jordan, $A$ nonlinear singularly perturbed Volterra integro-differential equation of nonconvolution type, Proc. Roy. Soc. Edinburgh, 80A (1978), 235-247.

[8] A. Kaye, Non-Newtonian Flow in Incompressible Fluids, Tech. Note 134, College of Aeronautics, Cranfield, England, 1962.

[9] A. S. Lodge, Elastic Liquids, Academic Press, London, New York, 1964.

[10] _ Body Tensor Fields in Continuum Mechanics, Academic Press, London, New York, 1974.

[11] A. S. Lodge, J. B. McLeod and J. A. Nohel, A nonlinear singularly perturbed Volterra integrodifferential equation occurring in polymer rheology, Proc. Roy. Soc. Edinburgh, 80A (1978), 99-137.

[12] P. Markowich and M. Renardy, A nonlinear Volterra integrodifferential equation describing the stretching of polymeric liquids, SIAM J. Math. Anal., 14 (1983), 66-97.

[13] R. K. Miller, Nonlinear Volterra Integral Equations, W. A. Benjamin, Menlo Park, 1971.

[14] O. Nevanlinna, Numerical solution of a singularly perturbed nonlinear Volterra equation, MRC TSR \# 1881, Univ. of Wisconsin, Madison, 1978.

[15] C. S. J. Petrie, Elongational Flows, Pitman Res. Notes in Math. 29, London, San Francisco, Melbourne, 1979.

[16] M. Renardy, A class of quasilinear parabolic equations with infinite delay and application to a problem in viscoelasticity, J. Differential Equations, 48 (1983), 280-292.

[17] M. Renardy, W. J. Hrusa and J. A. Nohel, Mathematical Problems in Viscoelasticity, Longman Scientific and Technical/John Wiley and Sons, Inc., New York, 1987.

[18] J. T. Schwartz, Nonlinear Functional Analysis, Gordon and Breach, New York, London, Paris, 1969. 
[19] C. A. Truesdell and W. Noll, The non-linear field theories of mechanics, In: S. Flügge (ed.): Handbuch der Physik II/3, Springer, Berlin, Heidelberg, New York, 1965.

[20] A. F. H. Ward and G. M. Jenkins, Normal thrust in dynamic torsion for rubberlike materials, Rheol. Acta, 1 (1958), 110-114.

[21] L. J. Zapas, Viscoelastic behavior under large deformations, J. Res. Nat. Bur. Stand., 70A (1966), 525-532.

Received July 25, 1989. This research was supported by the National Science Foundation under grant \# DMS 8601762.

GEORGETOWN UNIVERSITY

WASHINGTON, DC 20057 


\section{PACIFIC JOURNAL OF MATHEMATICS EDITORS}

\author{
V. S. VARADARAJAN \\ (Managing Editor) \\ University of California \\ Los Angeles, CA 90024-1555-05 \\ Herbert Clemens \\ University of Utah \\ Salt Lake City, UT 84112 \\ THOMAS ENRIGHT \\ University of California, San Diego \\ La Jolla, CA 92093
}

R. FINN

Stanford University

Stanford, CA 94305

HeRmann FlaschKa

University of Arizona

Tucson, AZ 85721

VAUGHAN F. R. JoNES

University of California

Berkeley, CA 94720

STEVEN KERCKHOFF

Stanford University

Stanford, CA 94305
C. C. MOORE

University of California

Berkeley, CA 94720

MaRTIN SCHARLEMANN

University of California

Santa Barbara, CA 93106

HAROLD STARK

University of California, San Diego

La Jolla, CA 92093

\section{ASSOCIATE EDITORS}
R. ARENS
E. F. BECKENBACH
B. H. NeumanN
F. WolF
K. YosHIDA

\section{SUPPORTING INSTITUTIONS}

UNIVERSITY OF ARIZONA

UNIVERSITY OF BRITISH COLUMBIA

CALIFORNIA INSTITUTE OF TECHNOLOGY

UNIVERSITY OF CALIFORNIA

MONTANA STATE UNIVERSITY

UNIVERSITY OF NEVADA, RENO

NEW MEXICO STATE UNIVERSITY

OREGON STATE UNIVERSITY
UNIVERSITY OF OREGON

UNIVERSITY OF SOUTHERN CALIFORNIA

STANFORD UNIVERSITY

UNIVERSITY OF HAWAII

UNIVERSITY OF TOKYO

UNIVERSITY OF UTAH

WASHINGTON STATE UNIVERSITY

UNIVERSITY OF WASHINGTON 


\section{Pacific Journal of Mathematics}

Vol. 149, No. $1 \quad$ May, 1991

Takao Akahori and Harunori Ameku, On the Romanov kernel and Kuranishi's $L^{2}$-estimate for $\bar{\partial}_{\mathrm{b}}$ over a ball in the strongly pseudo convex

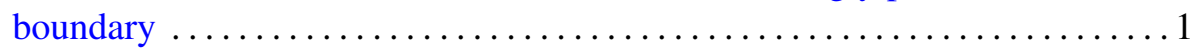

Robert Emile Beaudoin, The proper forcing axiom and stationary set

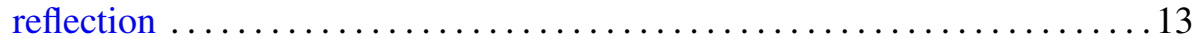

Hans Engler, A matrix Volterra integrodifferential equation occurring in

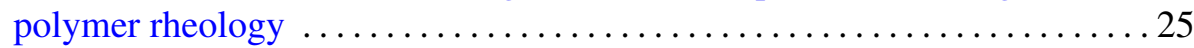

Zhong Ge, On a constrained variational problem and the spaces of

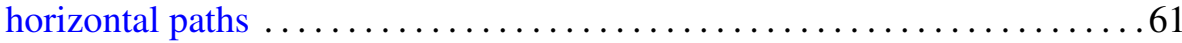

Yutaka Hemmi, Higher homotopy commutativity of $H$-spaces and the

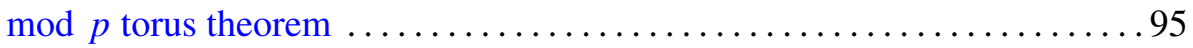

G. D. Johnson, An intrinsic characterization of a class of minimal surfaces

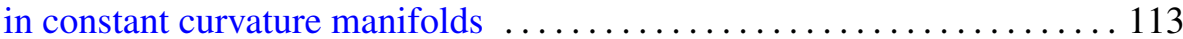

Min Ho Lee, Conjugates of equivariant holomorphic maps of symmetric domains

Jacek Nikiel, H. Murat Tuncali and Edward D. Tymchatyn, On the rim-structure of continuous images of ordered compacta

Tara Lynn Smith, Generalized Clifford-Littlewood-Eckmann groups . . . . . 157

Tara Lynn Smith, Generalized Clifford-Littlewood-Eckmann groups II:

Linear representations and applications 185 